Supporting Information

\title{
Charge-Switchable Polymeric Coating Kills Bacteria and Prevents Biofilm Formation In vivo
}

Jiaul Hoque ${ }^{1}$, Sreyan Ghosh ${ }^{1}$, Krishnamoorthy Paramanandham ${ }^{2}$ and Jayanta Haldar ${ }^{1,3 *}$

${ }^{1}$ Antimicrobial Research Laboratory, New Chemistry Unit, Jawaharlal Nehru Centre for Advanced Scientific Research, Jakkur, Bengaluru 560064, India

${ }^{2}$ National Institute of Veterinary Epidemiology and Disease Informatics (NIVEDI) Ramagondanahalli, Yelahanka, Bengaluru 560064, India

${ }^{3}$ School of Advanced Materials, Jawaharlal Nehru Centre for Advanced Scientific Research, Jakkur, Bengaluru 560064, India 


\section{Characterization data of compounds/polymers:}

$N$-hexadecyl-2-bromoethanamide: FT-IR $(\overline{\boldsymbol{v}}): 3255 \mathrm{~cm}^{-1}$ (amide $\mathrm{N}-\mathrm{H}$ str.), $2930 \mathrm{~cm}^{-1}\left(-\mathrm{CH}_{2}\right.$ assym. str.), 2850 ( $-\mathrm{CH}_{2}-$ sym. str.), $1680 \mathrm{~cm}^{-1}$ (Amide I, $\mathrm{C}=\mathrm{O}$ str.), $1565 \mathrm{~cm}^{-1}$ (Amide II, N$\mathrm{H}$ ben.), $1465 \mathrm{~cm}^{-1}\left(-\mathrm{CH}_{2}-\right.$ scissor); ${ }^{1} \mathrm{H}-\mathrm{NMR}$ : $\left(400 \mathrm{MHz}, \mathrm{CDCl}_{3}\right): \delta 0.879$ (t, terminal $-\mathrm{CH}_{3}$, $3 \mathrm{H}), 1.302\left(\mathrm{~m},-\left(\mathrm{CH}_{2}\right)_{13}-, 26 \mathrm{H}\right), 1.548\left(\mathrm{q},-\mathrm{CH}_{2}\left(\mathrm{CH}_{2}\right)_{13}-, 2 \mathrm{H}\right), 3.280$ (t, $\left.-\mathrm{CONHCH}_{2}-, 2 \mathrm{H}\right)$, 3.890 (s, $\left.-\mathrm{COCH}_{2} \mathrm{Br}, 2 \mathrm{H}\right), 6.578$ (br s, amide $\left.-\mathrm{NHCO}, 2 \mathrm{H}\right) ;{ }^{13} \mathrm{C}$ NMR $\left(100 \mathrm{MHz}, \mathrm{CDCl}_{3}\right.$ ): $\delta$ $14.22,22.98,26.89,29.28,29.40,29.52,29.58,29.70,29.79,32.11,40.41,165.66$.

$N$-hexadecyl-2-bromoethanoate: FT-IR $(\bar{v}): 2930 \mathrm{~cm}^{-1}\left(-\mathrm{CH}_{2}\right.$-assym. str.), $2850\left(-\mathrm{CH}_{2}-\right.$ sym. str.), $1740 \mathrm{~cm}^{-1}$ (C=O str. of ester), $1468 \mathrm{~cm}^{-1}$ (- $\mathrm{CH}_{2}-$ scissor); ${ }^{1} \mathrm{H}-\mathrm{NMR}$ : (400 MHz, $\left.\mathrm{CDCl}_{3}\right)$ : $\delta 0.889$ (t, terminal $\left.-\mathrm{CH}_{3}, 3 \mathrm{H}\right), 1.298\left(\mathrm{~m},-\left(\mathrm{CH}_{2}\right)_{13}-, 26 \mathrm{H}\right), 1.550\left(\mathrm{q},-\mathrm{CH}_{2}\left(\mathrm{CH}_{2}\right)_{13}-, 2 \mathrm{H}\right), 3.680$ $\left(\mathrm{t},-\mathrm{COOCH}_{2}-, 2 \mathrm{H}\right), 4.290\left(\mathrm{~s},-\mathrm{COCH}_{2} \mathrm{Br}, 2 \mathrm{H}\right),{ }^{13} \mathrm{C} \mathrm{NMR}\left(100 \mathrm{MHz}, \mathrm{CDCl}_{3}\right): \delta 14.19,22.76$, 26.903, 29.33, 29.41, 29.57, 29.63, 29.71, 29.81, 32.09, 40.41, 177.56.

1a: FT-IR ( $\bar{v})$ : $3200-3450 \mathrm{~cm}^{-1}$ (amide $\mathrm{N}-\mathrm{H}$ str.), $2930 \mathrm{~cm}^{-1}\left(-\mathrm{CH}_{2}-\right.$ assym. str.), $2860 \mathrm{~cm}^{-1}$ ( $-\mathrm{CH}_{2}-$ sym. str.), $1680 \mathrm{~cm}^{-1}$ (amide I, $\mathrm{C}=\mathrm{O}$ str.), $1555 \mathrm{~cm}^{-1}$ (amide II, $\mathrm{N}-\mathrm{H}$ ben.), $1470 \mathrm{~cm}^{-1}$ $\left(-\mathrm{CH}_{2}-\right.$ scissor); ${ }^{1} \mathrm{H}-\mathrm{NMR}\left(400 \mathrm{MHz}, \mathrm{CDCl}_{3}\right): \delta 0.857$ (t, terminal $\left.-\mathrm{CH}_{3}, 3 \mathrm{H}\right), 1.232(-$ $\left.\mathrm{CH}_{3}\left(\mathrm{CH}_{2}\right)_{13} \mathrm{CH}_{2-}, \quad 26 \mathrm{H}\right), \quad 1.512 \quad\left(\mathrm{CH}_{3}\left(\mathrm{CH}_{2}\right)_{13} \mathrm{CH}_{2} \mathrm{CH}_{2-}, \quad 2 \mathrm{H}\right), \quad 3.188 \quad$ ($\left.\left(\mathrm{CH}_{3}\right) \mathrm{N}^{+}\left(\mathrm{CH}_{2} \mathrm{CH}_{2}\right)_{2}\left(\mathrm{CH}_{2} \mathrm{CONH}\right)-, 3 \mathrm{H}\right), 3.483-3.586\left(-\left(\mathrm{CH}_{3}\right) \mathrm{N}^{+}\left(\mathrm{CH}_{2} \mathrm{CH}_{2}\right)_{2}\left(\mathrm{CH}_{2} \mathrm{CONH}\right)-, 4 \mathrm{H}\right)$, $3.844\left(-\mathrm{CH}_{2} \mathrm{CONHCH}_{2} \mathrm{CH}_{2}-, 2 \mathrm{H}\right), 4.476\left(-\left(\mathrm{CH}_{3}\right) \mathrm{N}^{+}\left(\mathrm{CH}_{2} \mathrm{CH}_{2}\right)_{2}\left(\mathrm{CH}_{2} \mathrm{CONH}\right)-, 2 \mathrm{H}\right), 8.318(-$ $\left.\mathrm{CH}_{2} \mathrm{CONHCH}_{2} \mathrm{CH}_{2-}, 1 \mathrm{H}\right) ;{ }^{13} \mathrm{C} \mathrm{NMR}(\mathrm{CP}-\mathrm{MAS}, 100 \mathrm{MHz}): \delta 14.75,24.44,30.52,32.87,40.32$, $46.57,52.92,57.14,66.49,163.69$.

1b: FT-IR ( $\bar{v})$ : $3200-3450 \mathrm{~cm}^{-1}$ (amide $\mathrm{N}-\mathrm{H}$ str.), $2932 \mathrm{~cm}^{-1}\left(-\mathrm{CH}_{2}-\right.$ assym. str.), $2865 \mathrm{~cm}^{-1}$ ( $-\mathrm{CH}_{2}-$ sym. str.), $1678 \mathrm{~cm}^{-1}$ (amide I, C=O str.), $1553 \mathrm{~cm}^{-1}$ (amide II, $\mathrm{N}-\mathrm{H}$ ben.), $1468 \mathrm{~cm}^{-1}$ $\left(-\mathrm{CH}_{2}-\right.$ scissor); ${ }^{1} \mathrm{H}-\mathrm{NMR}\left(400 \mathrm{MHz}, \mathrm{CDCl}_{3}\right): \delta 0.865$ (t, terminal $\left.-\mathrm{CH}_{3}, 3 \mathrm{H}\right), 1.238(-$ $\left.\mathrm{CH}_{3}\left(\mathrm{CH}_{2}\right)_{13} \mathrm{CH}_{2}-, \quad 26 \mathrm{H}\right), \quad 1.526 \quad\left(\mathrm{CH}_{3}\left(\mathrm{CH}_{2}\right)_{13} \mathrm{CH}_{2} \mathrm{CH}_{2-}, \quad 2 \mathrm{H}\right), \quad 3.202$ $\left.\left(\mathrm{CH}_{3}\right) \mathrm{N}^{+}\left(\mathrm{CH}_{2} \mathrm{CH}_{2}\right)_{2}\left(\mathrm{CH}_{2} \mathrm{CONH}\right)-, 3 \mathrm{H}\right), 3.497-3.602\left(-\left(\mathrm{CH}_{3}\right) \mathrm{N}^{+}\left(\mathrm{CH}_{2} \mathrm{CH}_{2}\right)_{2}\left(\mathrm{CH}_{2} \mathrm{CONH}\right)-, 4 \mathrm{H}\right)$, 3.877-3.994 $\left(-\mathrm{CH}_{2} \mathrm{CONHCH}_{2} \mathrm{CH}_{2}-, 2 \mathrm{H}\right), 4.503\left(-\left(\mathrm{CH}_{3}\right) \mathrm{N}^{+}\left(\mathrm{CH}_{2} \mathrm{CH}_{2}\right)_{2}\left(\mathrm{CH}_{2} \mathrm{CONH}\right)-, 2 \mathrm{H}\right)$, $8.331\left(-\mathrm{CH}_{2} \mathrm{CONHCH}_{2} \mathrm{CH}_{2}-, 1 \mathrm{H}\right) ;{ }^{13} \mathrm{C}$ NMR (CP-MAS, $\left.100 \mathrm{MHz}\right): \delta 14.70,24.34,30.52$, $32.78,40.30,46.54,52.92,57.24,66.42,163.59$.

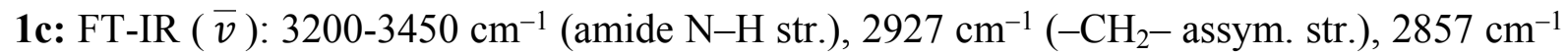
( $-\mathrm{CH}_{2}-$ sym. str.), $1676 \mathrm{~cm}^{-1}$ (amide I, C=O str.), $1550 \mathrm{~cm}^{-1}$ (amide II, $\mathrm{N}-\mathrm{H}$ ben.), $1469 \mathrm{~cm}^{-1}$ (- $\mathrm{CH}_{2}-$ scissor); ${ }^{1} \mathrm{H}-\mathrm{NMR}\left(400 \mathrm{MHz}, \mathrm{CDCl}_{3}\right): \delta 0.868$ (t, terminal $\left.-\mathrm{CH}_{3}, 3 \mathrm{H}\right), 1.244(-$ $\left.\mathrm{CH}_{3}\left(\mathrm{CH}_{2}\right)_{13} \mathrm{CH}_{2}-, \quad 26 \mathrm{H}\right), \quad 1.534 \quad\left(\mathrm{CH}_{3}\left(\mathrm{CH}_{2}\right)_{13} \mathrm{CH}_{2} \mathrm{CH}_{2-}, \quad 2 \mathrm{H}\right), \quad 3.214 \quad$ ($\left.\left(\mathrm{CH}_{3}\right) \mathrm{N}^{+}\left(\mathrm{CH}_{2} \mathrm{CH}_{2}\right)_{2}\left(\mathrm{CH}_{2} \mathrm{CONH}\right)-, 3 \mathrm{H}\right), 3.504-3.602\left(-\left(\mathrm{CH}_{3}\right) \mathrm{N}^{+}\left(\mathrm{CH}_{2} \mathrm{CH}_{2}\right)_{2}\left(\mathrm{CH}_{2} \mathrm{CONH}\right)-\right.$, $4 \mathrm{H}), \quad 3.877-4.095 \quad\left(-\mathrm{CH}_{2} \mathrm{CONHCH}_{2} \mathrm{CH}_{2}-, \quad 2 \mathrm{H}\right), \quad 4.518-4.719$ $\left.\left(\mathrm{CH}_{3}\right) \mathrm{N}^{+}\left(\mathrm{CH}_{2} \mathrm{CH}_{2}\right)_{2}\left(\mathrm{CH}_{2} \mathrm{CONH}\right)-, 2 \mathrm{H}\right), 8.318\left(-\mathrm{CH}_{2} \mathrm{CONHCH}_{2} \mathrm{CH}_{2}-, 1 \mathrm{H}\right) ;{ }^{13} \mathrm{C} \mathrm{NMR}(\mathrm{CP}-$ MAS, $100 \mathrm{MHz}): \delta 14.65,23.49,30.42,32.77,40.39,46.67,51.22,57.04,66.45,163.58$.

2a: FT-IR ( $\bar{v})$ : $2920 \mathrm{~cm}^{-1}$ (- $\mathrm{CH}_{2}-$ assym. str.), $2860 \mathrm{~cm}^{-1}$ ( $-\mathrm{CH}_{2}-$ sym. str.), $1750 \mathrm{~cm}^{-1}(-\mathrm{C}=\mathrm{O}$ str.), $1470 \mathrm{~cm}^{-1}\left(-\mathrm{CH}_{2}-\right.$ scissor); ${ }^{1} \mathrm{H}-\mathrm{NMR}\left(400 \mathrm{MHz}, \mathrm{CDCl}_{3}\right): \delta 0.855$ (t, terminal $\left.-\mathrm{CH}_{3}, 3 \mathrm{H}\right)$, $1.234 \quad\left(-\mathrm{CH}_{3}\left(\mathrm{CH}_{2}\right)_{13} \mathrm{CH}_{2}-, \quad 26 \mathrm{H}\right), \quad 1.655 \quad\left(\mathrm{CH}_{3}\left(\mathrm{CH}_{2}\right)_{13} \mathrm{CH}_{2} \mathrm{CH}_{2}-, \quad 2 \mathrm{H}\right), \quad 3.584-3.618 \quad$ ($\left.\left(\mathrm{CH}_{3}\right) \mathrm{N}^{+}\left(\mathrm{CH}_{2} \mathrm{CH}_{2}\right)_{2}\left(\mathrm{CH}_{2} \mathrm{COOCH}_{2}\right)-, 3 \mathrm{H}\right), 4.177\left(-\left(\mathrm{CH}_{3}\right) \mathrm{N}^{+}\left(\mathrm{CH}_{2} \mathrm{CH}_{2}\right)_{2}\left(\mathrm{CH}_{2} \mathrm{COOCH}_{2}\right)-\right.$ and ($\left.\mathrm{CH}_{2} \mathrm{COOCH}_{2} \mathrm{CH}_{2}-, 6 \mathrm{H}\right), 4.704\left(-\left(\mathrm{CH}_{3}\right) \mathrm{N}^{+}\left(\mathrm{CH}_{2} \mathrm{CH}_{2}\right)_{2}\left(\mathrm{CH}_{2} \mathrm{COOCH}_{2}\right)-, 2 \mathrm{H}\right) ;{ }^{13} \mathrm{C} \mathrm{NMR}(\mathrm{CP}-$ MAS, $100 \mathrm{MHz}): \delta 14.89,24.55,27.25,27.82,32.86,46.26,46.86,47.51,52.40,53.04,53.82$, $60.07,61.83,66.37,165.17$. 
2b: FT-IR ( $\bar{v}$ ): $2923 \mathrm{~cm}^{-1}$ (- $\mathrm{CH}_{2}-$ assym. str.), $2858 \mathrm{~cm}^{-1}$ ( $-\mathrm{CH}_{2}-$ sym. str.), $1750 \mathrm{~cm}^{-1}(-\mathrm{C}=\mathrm{O}$ str.), $1472 \mathrm{~cm}^{-1}\left(-\mathrm{CH}_{2}-\right.$ scissor); ${ }^{1} \mathrm{H}-\mathrm{NMR}\left(400 \mathrm{MHz}, \mathrm{CDCl}_{3}\right): \delta 0.851\left(\mathrm{t}\right.$, terminal $\left.-\mathrm{CH}_{3}, 3 \mathrm{H}\right)$, $1.230 \quad\left(-\mathrm{CH}_{3}\left(\mathrm{CH}_{2}\right)_{13} \mathrm{CH}_{2}-, \quad 26 \mathrm{H}\right), \quad 1.647 \quad\left(\mathrm{CH}_{3}\left(\mathrm{CH}_{2}\right)_{13} \mathrm{CH}_{2} \mathrm{CH}_{2-}, \quad 2 \mathrm{H}\right), \quad 3.579-3.663$ $\left.\left(\mathrm{CH}_{3}\right) \mathrm{N}^{+}\left(\mathrm{CH}_{2} \mathrm{CH}_{2}\right)_{2}\left(\mathrm{CH}_{2} \mathrm{COOCH}_{2}\right)-, 3 \mathrm{H}\right), 4.166\left(-\left(\mathrm{CH}_{3}\right) \mathrm{N}^{+}\left(\mathrm{CH}_{2} \mathrm{CH}_{2}\right)_{2}\left(\mathrm{CH}_{2} \mathrm{COOCH}_{2}\right)-\right.$ and $(-$ $\left.\mathrm{CH}_{2} \mathrm{COOCH}_{2} \mathrm{CH}_{2}-, 6 \mathrm{H}\right), 4.698\left(-\left(\mathrm{CH}_{3}\right) \mathrm{N}^{+}\left(\mathrm{CH}_{2} \mathrm{CH}_{2}\right)_{2}\left(\mathrm{CH}_{2} \mathrm{COOCH}_{2}\right)-, 2 \mathrm{H}\right) ;{ }^{13} \mathrm{C} \mathrm{NMR}(\mathrm{CP}-$ MAS, $100 \mathrm{MHz}): \delta 14.9,24.52,27.18,30.66,32.84,34.22,35.74,46.98,52.53,53.58,54.36$, $62.05,66.19,165.32$.

2c: FT-IR ( $\bar{v}): 2918 \mathrm{~cm}^{-1}$ (- $\mathrm{CH}_{2}-$ assym. str.), $2855 \mathrm{~cm}^{-1}$ ( $-\mathrm{CH}_{2}-$ sym. str.), $1747 \mathrm{~cm}^{-1}(-\mathrm{C}=\mathrm{O}$ str.), $1467 \mathrm{~cm}^{-1}\left(-\mathrm{CH}_{2}-\right.$ scissor); ${ }^{1} \mathrm{H}-\mathrm{NMR}\left(400 \mathrm{MHz}, \mathrm{CDCl}_{3}\right): \delta 0.830\left(\mathrm{t}\right.$, terminal $\left.-\mathrm{CH}_{3}, 3 \mathrm{H}\right)$, $1.209 \quad\left(-\mathrm{CH}_{3}\left(\mathrm{CH}_{2}\right)_{13} \mathrm{CH}_{2}-, 26 \mathrm{H}\right), \quad 1.623\left(\mathrm{CH}_{3}\left(\mathrm{CH}_{2}\right)_{13} \mathrm{CH}_{2} \mathrm{CH}_{2}-, 2 \mathrm{H}\right), \quad 3.564-3.605$ $\left.\left(\mathrm{CH}_{3}\right) \mathrm{N}^{+}\left(\mathrm{CH}_{2} \mathrm{CH}_{2}\right)_{2}\left(\mathrm{CH}_{2} \mathrm{COOCH}_{2}\right)-, 3 \mathrm{H}\right), 3.654-4.143\left(-\left(\mathrm{CH}_{3}\right) \mathrm{N}^{+}\left(\mathrm{CH}_{2} \mathrm{CH}_{2}\right)_{2}\left(\mathrm{CH}_{2} \mathrm{COOCH}_{2}\right)-\right.$ and $\left(-\mathrm{CH}_{2} \mathrm{COOCH}_{2} \mathrm{CH}_{2}-, 6 \mathrm{H}\right), 4.699\left(-\left(\mathrm{CH}_{3}\right) \mathrm{N}^{+}\left(\mathrm{CH}_{2} \mathrm{CH}_{2}\right)_{2}\left(\mathrm{CH}_{2} \mathrm{COOCH}_{2}\right)-, 2 \mathrm{H}\right), ;{ }^{13} \mathrm{C} \mathrm{NMR}$ (CP-MAS, $100 \mathrm{MHz}$ ): $\delta$ 14.89, 24.55, 27.25, 27.82, 32.86, 46.26, 46.86, 47.51, 52.40, 53.04, $53.82,60.07,61.83,66.37,165.17$.

3a: FT-IR ( $\bar{v})$ : 3200-3450 $\mathrm{cm}^{-1}$ (amide $\mathrm{N}-\mathrm{H}$ str.), $2925 \mathrm{~cm}^{-1}\left(-\mathrm{CH}_{2}-\right.$ assym. str.), $2867 \mathrm{~cm}^{-1}$ ( $-\mathrm{CH}_{2}-$ sym. str.), $1677 \mathrm{~cm}^{-1}$ (amide I, C=O str.), $1550 \mathrm{~cm}^{-1}$ (amide II, $\mathrm{N}-\mathrm{H}$ ben.), $1467 \mathrm{~cm}^{-1}$ $\left(-\mathrm{CH}_{2}-\right.$ scissor); ${ }^{1} \mathrm{H}-\mathrm{NMR}\left(400 \mathrm{MHz}, \mathrm{CDCl}_{3}\right): \delta 0.879$ (t, terminal $\left.-\mathrm{CH}_{3}, 3 \mathrm{H}\right), 1.256(-$ $\left.\mathrm{CH}_{3}\left(\mathrm{CH}_{2}\right)_{13} \mathrm{CH}_{2-}, \quad 26 \mathrm{H}\right), \quad 1.546 \quad\left(\mathrm{CH}_{3}\left(\mathrm{CH}_{2}\right)_{13} \mathrm{CH}_{2} \mathrm{CH}_{2-}, \quad 2 \mathrm{H}\right), \quad 3.228 \quad$ ($\left.\left(\mathrm{CH}_{3}\right) \mathrm{N}^{+}\left(\mathrm{CH}_{2} \mathrm{CH}_{2}\right)_{2}\left(\mathrm{CH}_{2} \mathrm{CONH}\right)-, 3 \mathrm{H}\right), 3.882-3.963\left(-\left(\mathrm{CH}_{3}\right) \mathrm{N}^{+}\left(\mathrm{CH}_{2} \mathrm{CH}_{2}\right)_{2}\left(\mathrm{CH}_{2} \mathrm{CONH}\right)-, 4 \mathrm{H}\right)$, $4.563\left(-\mathrm{CH}_{2} \mathrm{CONHCH}_{2} \mathrm{CH}_{2}-, 2 \mathrm{H}\right), 5.004\left(-\left(\mathrm{CH}_{3}\right) \mathrm{N}^{+}\left(\mathrm{CH}_{2} \mathrm{CH}_{2}\right)_{2}\left(\mathrm{CH}_{2} \mathrm{CONH}\right)-, 2 \mathrm{H}\right), 8.312$ ($\left.\mathrm{CH}_{2} \mathrm{CONHCH} \mathrm{CH}_{2}-, 1 \mathrm{H}\right) ;{ }^{13} \mathrm{C} \mathrm{NMR}(\mathrm{CP}-\mathrm{MAS}, 100 \mathrm{MHz}): \delta 14.71,24.50,30.53,32.80,40.42$, 46.63, 52.85, 57.17, 66.49, 163.65.

3b: FT-IR ( $\bar{v}$ ): 3200-3450 cm-1 (amide N-H str.), $2927 \mathrm{~cm}^{-1}$ (- $\mathrm{CH}_{2}-$ assym. str.), $2857 \mathrm{~cm}^{-1}$ ( $-\mathrm{CH}_{2}-$ sym. str.), $1675 \mathrm{~cm}^{-1}$ (amide I, $\mathrm{C}=\mathrm{O}$ str.), $1560 \mathrm{~cm}^{-1}$ (amide II, $\mathrm{N}-\mathrm{H}$ ben.), $1474 \mathrm{~cm}^{-1}$ $\left(-\mathrm{CH}_{2}-\right.$ scissor); ${ }^{1} \mathrm{H}-\mathrm{NMR}\left(400 \mathrm{MHz}, \mathrm{CDCl}_{3}\right): \delta 0.880$ (t, terminal $\left.-\mathrm{CH}_{3}, 3 \mathrm{H}\right), 1.256(-$ $\left.\mathrm{CH}_{3}\left(\mathrm{CH}_{2}\right)_{13} \mathrm{CH}_{2}-, \quad 26 \mathrm{H}\right), \quad 1.549 \quad\left(\mathrm{CH}_{3}\left(\mathrm{CH}_{2}\right)_{13} \mathrm{CH}_{2} \mathrm{CH}_{2}, \quad, \quad 2 \mathrm{H}\right), \quad 3.231 \quad$ ($\left.\left(\mathrm{CH}_{3}\right) \mathrm{N}^{+}\left(\mathrm{CH}_{2} \mathrm{CH}_{2}\right)_{2}\left(\mathrm{CH}_{2} \mathrm{CONH}\right)-, \quad 3 \mathrm{H}\right), 3.976\left(-\left(\mathrm{CH}_{3}\right) \mathrm{N}^{+}\left(\mathrm{CH}_{2} \mathrm{CH}_{2}\right)_{2}\left(\mathrm{CH}_{2} \mathrm{CONH}\right)-, 4 \mathrm{H}\right)$, 4.361-4.579 (- $\left.\mathrm{CH}_{2} \mathrm{CONHCH}_{2} \mathrm{CH}_{2}-, 2 \mathrm{H}\right), 5.028\left(-\left(\mathrm{CH}_{3}\right) \mathrm{N}^{+}\left(\mathrm{CH}_{2} \mathrm{CH}_{2}\right)_{2}\left(\mathrm{CH}_{2} \mathrm{CONH}\right)-, 2 \mathrm{H}\right)$, $8.307\left(-\mathrm{CH}_{2} \mathrm{CONHCH}_{2} \mathrm{CH}_{2}-, 1 \mathrm{H}\right) ;{ }^{13} \mathrm{C}$ NMR (CP-MAS, $\left.100 \mathrm{MHz}\right): \delta 14.66,24.46,30.60$, $32.81,40.35,46.60,52.82,57.21,66.33,163.65$.

3c: FT-IR ( $\bar{v}$ ): 3200-3450 cm $\mathrm{cm}^{-1}$ (amide $\mathrm{N}-\mathrm{H}$ str.), $2940 \mathrm{~cm}^{-1}\left(-\mathrm{CH}_{2}-\right.$ assym. str.), $2853 \mathrm{~cm}^{-1}$ ( $-\mathrm{CH}_{2}-$ sym. str.), $1671 \mathrm{~cm}^{-1}$ (amide I, C=O str.), $1551 \mathrm{~cm}^{-1}$ (amide II, $\mathrm{N}-\mathrm{H}$ ben.), $1460 \mathrm{~cm}^{-1}$ $\left(-\mathrm{CH}_{2}-\right.$ scissor); ${ }^{1} \mathrm{H}-\mathrm{NMR}\left(400 \mathrm{MHz}, \mathrm{CDCl}_{3}\right): \delta 0.880$ (t, terminal $\left.-\mathrm{CH}_{3}, 3 \mathrm{H}\right), 1.284(-$ $\left.\mathrm{CH}_{3}\left(\mathrm{CH}_{2}\right)_{13} \mathrm{CH}_{2}-, \quad 26 \mathrm{H}\right), \quad 1.551 \quad\left(\mathrm{CH}_{3}\left(\mathrm{CH}_{2}\right)_{13} \mathrm{CH}_{2} \mathrm{CH}_{2-}, \quad 2 \mathrm{H}\right), \quad 3.229 \quad$ ($\left.\left(\mathrm{CH}_{3}\right) \mathrm{N}^{+}\left(\mathrm{CH}_{2} \mathrm{CH}_{2}\right)_{2}\left(\mathrm{CH}_{2} \mathrm{CONH}\right)-, 3 \mathrm{H}\right), 3.835-3.976\left(-\left(\mathrm{CH}_{3}\right) \mathrm{N}^{+}\left(\mathrm{CH}_{2} \mathrm{CH}_{2}\right)_{2}\left(\mathrm{CH}_{2} \mathrm{CONH}\right)-, 4 \mathrm{H}\right)$, $4.600\left(-\mathrm{CH}_{2} \mathrm{CONHCH} \mathrm{CH}_{2}-, 2 \mathrm{H}\right), 5.025-5.046\left(-\left(\mathrm{CH}_{3}\right) \mathrm{N}^{+}\left(\mathrm{CH}_{2} \mathrm{CH}_{2}\right)_{2}\left(\mathrm{CH}_{2} \mathrm{CONH}\right)-, 2 \mathrm{H}\right)$, $8.329\left(-\mathrm{CH}_{2} \mathrm{CONHCH}_{2} \mathrm{CH}_{2}-, 1 \mathrm{H}\right) ;{ }^{13} \mathrm{C} \mathrm{NMR}$ (CP-MAS, $\left.100 \mathrm{MHz}\right): \delta 14.89,24.51,32.90$, $40.60,46.35,53.13,56.54,56.83,66.39,164.77$.

4a: FT-IR ( $\bar{v})$ : $2920 \mathrm{~cm}^{-1}$ (- $\mathrm{CH}_{2}-$ assym. str.), $2863 \mathrm{~cm}^{-1}$ (- $\mathrm{CH}_{2}-$ sym. str.), $1750 \mathrm{~cm}^{-1}(-\mathrm{C}=\mathrm{O}$ str.), $1460 \mathrm{~cm}^{-1}\left(-\mathrm{CH}_{2}-\right.$ scissor); ${ }^{1} \mathrm{H}-\mathrm{NMR}\left(400 \mathrm{MHz}, \mathrm{CDCl}_{3}\right): \delta 0.880\left(\mathrm{t}\right.$, terminal $\left.-\mathrm{CH}_{3}, 3 \mathrm{H}\right)$, $1.259\left(-\mathrm{CH}_{3}\left(\mathrm{CH}_{2}\right)_{13} \mathrm{CH}_{2}-, 26 \mathrm{H}\right), 1.696-1.773\left(\mathrm{CH}_{3}\left(\mathrm{CH}_{2}\right)_{13} \mathrm{CH}_{2} \mathrm{CH}_{2}-, 2 \mathrm{H}\right), 3.954-4.114(-$ $\left(\mathrm{CH}_{3}\right) \mathrm{N}^{+}\left(\mathrm{CH}_{2} \mathrm{CH}_{2}\right)_{2}\left(\mathrm{CH}_{2} \mathrm{COOCH}_{2}\right)-$ and $\left(-\left(\mathrm{CH}_{3}\right) \mathrm{N}^{+}\left(\mathrm{CH}_{2} \mathrm{CH}_{2}\right)_{2}\left(\mathrm{CH}_{2} \mathrm{COOCH}_{2}\right)-, 7 \mathrm{H}\right), 4.689-$ $4.888\left(\left(-\left(\mathrm{CH}_{3}\right) \mathrm{N}^{+}\left(\mathrm{CH}_{2} \mathrm{CH}_{2}\right)_{2}\left(\mathrm{CH}_{2} \mathrm{COOCH}_{2}\right)-\right.\right.$ and $\left.-\mathrm{CH}_{2} \mathrm{COOCH}_{2} \mathrm{CH}_{2}-, 4 \mathrm{H}\right) ;{ }^{13} \mathrm{C} \mathrm{NMR}(\mathrm{CP}-$ 
MAS, $100 \mathrm{MHz}): \delta 14.90,24.63,29.37,32.87,34.33,40.61,46.89,47.51,52.89,57.07,65.95$, 162.86.

4b: FT-IR ( $\bar{v})$ : $2920 \mathrm{~cm}^{-1}$ (- $\mathrm{CH}_{2}-$ assym. str.), $2863 \mathrm{~cm}^{-1}$ ( $-\mathrm{CH}_{2}-$ sym. str.), $1750 \mathrm{~cm}^{-1}$ (-C=O str.), $1460 \mathrm{~cm}^{-1}\left(-\mathrm{CH}_{2}-\right.$ scissor); ${ }^{1} \mathrm{H}-\mathrm{NMR}\left(400 \mathrm{MHz}, \mathrm{CDCl}_{3}\right): \delta 0.878\left(\mathrm{t}\right.$, terminal $\left.-\mathrm{CH}_{3}, 3 \mathrm{H}\right)$, $1.255\left(-\mathrm{CH}_{3}\left(\mathrm{CH}_{2}\right)_{13} \mathrm{CH}_{2}-, 26 \mathrm{H}\right), 1.678-1.689\left(\mathrm{CH}_{3}\left(\mathrm{CH}_{2}\right)_{13} \mathrm{CH}_{2} \mathrm{CH}_{2}-, 2 \mathrm{H}\right), 3.828-4.221$ ($\left(\mathrm{CH}_{3}\right) \mathrm{N}^{+}\left(\mathrm{CH}_{2} \mathrm{CH}_{2}\right)_{2}\left(\mathrm{CH}_{2} \mathrm{COOCH}_{2}\right)-$ and $\left(-\left(\mathrm{CH}_{3}\right) \mathrm{N}^{+}\left(\mathrm{CH}_{2} \mathrm{CH}_{2}\right)_{2}\left(\mathrm{CH}_{2} \mathrm{COOCH}_{2}\right)-, 7 \mathrm{H}\right), 4.676-$ $4.870\left(\left(-\left(\mathrm{CH}_{3}\right) \mathrm{N}^{+}\left(\mathrm{CH}_{2} \mathrm{CH}_{2}\right)_{2}\left(\mathrm{CH}_{2} \mathrm{COOCH}_{2}\right)-\right.\right.$ and $\left.-\mathrm{CH}_{2} \mathrm{COOCH}_{2} \mathrm{CH}_{2}-, 4 \mathrm{H}\right) ;{ }^{13} \mathrm{C} \mathrm{NMR}(\mathrm{CP}-$ MAS, $100 \mathrm{MHz}): \delta 14.90,24.69,29.35,32.87,34.33,40.61,46.89,47.41,52.89,57.07,65.93$, 163.86 .

4c: FT-IR ( $\bar{v})$ : $2910 \mathrm{~cm}^{-1}$ (-CH $2-$ assym. str.), $2863 \mathrm{~cm}^{-1}$ (- $\mathrm{CH}_{2}-$ sym. str.), $1750 \mathrm{~cm}^{-1}(-\mathrm{C}=\mathrm{O}$ str.), $1456 \mathrm{~cm}^{-1}\left(-\mathrm{CH}_{2}-\right.$ scissor); ${ }^{1} \mathrm{H}-\mathrm{NMR}\left(400 \mathrm{MHz}, \mathrm{CDCl}_{3}\right): \delta 0.877$ (t, terminal $\left.-\mathrm{CH}_{3}, 3 \mathrm{H}\right)$, $1.255 \quad\left(-\mathrm{CH}_{3}\left(\mathrm{CH}_{2}\right)_{13} \mathrm{CH}_{2}-, \quad 26 \mathrm{H}\right), \quad 1.690 \quad\left(\mathrm{CH}_{3}\left(\mathrm{CH}_{2}\right)_{13} \mathrm{CH}_{2} \mathrm{CH}_{2}-, \quad 2 \mathrm{H}\right), \quad 3.827-4.224 \quad$ ($\left(\mathrm{CH}_{3}\right) \mathrm{N}^{+}\left(\mathrm{CH}_{2} \mathrm{CH}_{2}\right)_{2}\left(\mathrm{CH}_{2} \mathrm{COOCH}_{2}\right)-$ and $\left(-\left(\mathrm{CH}_{3}\right) \mathrm{N}^{+}\left(\mathrm{CH}_{2} \mathrm{CH}_{2}\right)_{2}\left(\mathrm{CH}_{2} \mathrm{COOCH}_{2}\right)-, 7 \mathrm{H}\right), 4.752-$ $4.869\left(\left(-\left(\mathrm{CH}_{3}\right) \mathrm{N}^{+}\left(\mathrm{CH}_{2} \mathrm{CH}_{2}\right)_{2}\left(\mathrm{CH}_{2} \mathrm{COOCH}_{2}\right)-\right.\right.$ and $\left.-\mathrm{CH}_{2} \mathrm{COOCH}_{2} \mathrm{CH}_{2}-, 4 \mathrm{H}\right) ;{ }^{13} \mathrm{C} \mathrm{NMR}(\mathrm{CP}-$ MAS, $100 \mathrm{MHz}): \delta$ 14.90, 24.52, 27.18, 30.66, 32.84, 34.22, 35.74, 46.98. 52.53, 54.36.62.05,66.19 165.32.

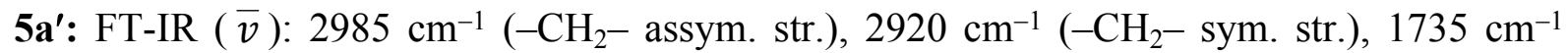
$\left(-\mathrm{C}=\mathrm{O}\right.$ str.), $1465 \mathrm{~cm}^{-1} \quad\left(-\mathrm{CH}_{2}-\right.$ scissor); ${ }^{1} \mathrm{H}-\mathrm{NMR}\left(400 \mathrm{MHz}, \mathrm{CDCl}_{3}\right): \delta 1.438$ (s, $\left.\mathrm{C}\left(\mathrm{CH}_{3}\right)_{3} \mathrm{OCO}-, 9 \mathrm{H}\right), 2.356-2.538\left(-\mathrm{N}\left(\mathrm{CH}_{2} \mathrm{CH}_{2}\right) \mathrm{N}-, 4 \mathrm{H}\right), 3.018-3.387\left(-\mathrm{CH}_{3}\right) \mathrm{N}^{+}\left(\mathrm{CH}_{2} \mathrm{CH}_{2}\right)_{2}-$, $\left.3 \mathrm{H}), 3.428-3.597-\left(\mathrm{CH}_{3}\right) \mathrm{N}^{+}\left(\mathrm{CH}_{2} \mathrm{CH}_{2}\right)_{2}-, 4 \mathrm{H}\right), 4.220-4.354\left(-\mathrm{CH}_{3}\right) \mathrm{N}^{+}\left(\mathrm{CH}_{2} \mathrm{CH}_{2}\right)_{2} \mathrm{CH}_{2} \mathrm{COO}-$, $2 \mathrm{H})$.

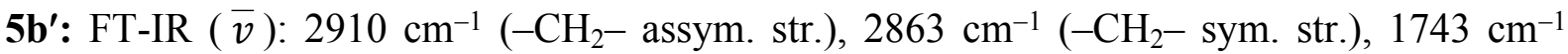
(-C=O str.), $1467 \mathrm{~cm}^{-1}$ (- $\mathrm{CH}_{2}-$ scissor); ${ }^{1} \mathrm{H}-\mathrm{NMR}\left(400 \mathrm{MHz}, \mathrm{CDCl}_{3}\right): \delta 1.508$ (s, $\left.\mathrm{C}\left(\mathrm{CH}_{3}\right)_{3} \mathrm{OCO}-, 9 \mathrm{H}\right), 2.350-2.479\left(-\mathrm{N}\left(\mathrm{CH}_{2} \mathrm{CH}_{2}\right) \mathrm{N}-, 4 \mathrm{H}\right), 3.028-3.382\left(-\mathrm{CH}_{3}\right) \mathrm{N}^{+}\left(\mathrm{CH}_{2} \mathrm{CH}_{2}\right)_{2}-$, $3 \mathrm{H})$, 3.428-3.597 - $\left.\left(\mathrm{CH}_{3}\right) \mathrm{N}^{+}\left(\mathrm{CH}_{2} \mathrm{CH}_{2}\right)_{2}-, 4 \mathrm{H}\right), 4.220-4.354\left(-\mathrm{CH}_{3}\right) \mathrm{N}^{+}\left(\mathrm{CH}_{2} \mathrm{CH}_{2}\right)_{2} \mathrm{CH}_{2} \mathrm{COO}-$, $2 \mathrm{H})$.

5c': FT-IR ( $\bar{v}): 2918 \mathrm{~cm}^{-1}$ (-CH $\mathrm{CH}_{2}-$ assym. str.), $2864 \mathrm{~cm}^{-1}\left(-\mathrm{CH}_{2}-\right.$ sym. str.), $1742 \mathrm{~cm}^{-1}$ $\left(-\mathrm{C}=\mathrm{O}\right.$ str.), $1465 \mathrm{~cm}^{-1} \quad\left(-\mathrm{CH}_{2}-\right.$ scissor); ${ }^{1} \mathrm{H}-\mathrm{NMR}\left(400 \mathrm{MHz}, \mathrm{CDCl}_{3}\right): \delta 1.498$ (s, $\left.\mathrm{C}\left(\mathrm{CH}_{3}\right)_{3} \mathrm{OCO}-, 9 \mathrm{H}\right), 2.298-2.491\left(-\mathrm{N}\left(\mathrm{CH}_{2} \mathrm{CH}_{2}\right) \mathrm{N}-, 4 \mathrm{H}\right), 3.118-3.401\left(-\mathrm{CH}_{3}\right) \mathrm{N}^{+}\left(\mathrm{CH}_{2} \mathrm{CH}_{2}\right)_{2}-$, $\left.\left.3 \mathrm{H}), 3.398-3.607-\left(\mathrm{CH}_{3}\right) \mathrm{N}^{+}\left(\mathrm{CH}_{2} \mathrm{CH}_{2}\right)_{2}-, 4 \mathrm{H}\right), 4.228-4.361\left(-\mathrm{CH}_{3}\right) \mathrm{N}^{+}\left(\mathrm{CH}_{2} \mathrm{CH}_{2}\right)_{2} \mathrm{CH}_{2} \mathrm{COO}-\right)$.

5a": FT-IR ( $\bar{v}$ ): 3200-3450 cm $\mathrm{cm}^{-1}$ (amide $\mathrm{N}-\mathrm{H}$ str.), $2940 \mathrm{~cm}^{-1}\left(-\mathrm{CH}_{2}-\right.$ assym. str.), $2853 \mathrm{~cm}^{-1}$ ( $-\mathrm{CH}_{2}$ - sym. str.), $1737 \mathrm{~cm}^{-1}$ ( $-\mathrm{C}=\mathrm{O}$ str.), $1671 \mathrm{~cm}^{-1}$ (amide I, $\mathrm{C}=\mathrm{O}$ str.), $1551 \mathrm{~cm}^{-1}$ (amide II, $\mathrm{N}-\mathrm{H}$ ben.), $1460 \mathrm{~cm}^{-1}$ (- $\mathrm{CH}_{2}-$ scissor); ${ }^{1} \mathrm{H}-\mathrm{NMR}\left(400 \mathrm{MHz}, \mathrm{CDCl}_{3}\right): \delta 0.880(\mathrm{t}$, terminal $\left.\mathrm{CH}_{3}, 3 \mathrm{H}\right), 1.284\left(-\mathrm{CH}_{3}\left(\mathrm{CH}_{2}\right)_{13} \mathrm{CH}_{2}-, 26 \mathrm{H}\right), 1.456\left(\mathrm{~s}, \mathrm{C}\left(\mathrm{CH}_{3}\right)_{3} \mathrm{OCO}-\right), 1.551\left(\mathrm{CH}_{3}\left(\mathrm{CH}_{2}\right)_{13} \mathrm{CH}_{2-}\right.$ $\left.\mathrm{CH}_{2}-, \quad 2 \mathrm{H}\right), \quad 3.229 \quad\left(-\left(\mathrm{CH}_{3}\right) \mathrm{N}^{+}\left(\mathrm{CH}_{2} \mathrm{CH}_{2}\right)_{2}\left(\mathrm{CH}_{2} \mathrm{CONH}\right)-, \quad 3 \mathrm{H}\right), \quad 3.835-3.976 \quad(-$ $\left.\left(\mathrm{CH}_{3}\right) \mathrm{N}^{+}\left(\mathrm{CH}_{2} \mathrm{CH}_{2}\right)_{2}\left(\mathrm{CH}_{2} \mathrm{CONH}\right)-, 4 \mathrm{H}\right), 4.600\left(-\mathrm{CH}_{2} \mathrm{CONHCH}_{2} \mathrm{CH}_{2}-, 2 \mathrm{H}\right)$, 5.025-5.046 ($\left.\left(\mathrm{CH}_{3}\right) \mathrm{N}^{+}\left(\mathrm{CH}_{2} \mathrm{CH}_{2}\right)_{2}\left(\mathrm{CH}_{2} \mathrm{CONH}\right)-, 2 \mathrm{H}\right), 8.329\left(-\mathrm{CH}_{2} \mathrm{CONHCH}_{2} \mathrm{CH}_{2}-, 1 \mathrm{H}\right)$.

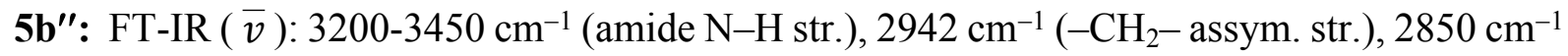
( $-\mathrm{CH}_{2}-$ sym. str.), $1735 \mathrm{~cm}^{-1}$ ( $-\mathrm{C}=\mathrm{O}$ str.), $1671 \mathrm{~cm}^{-1}$ (amide I, $\mathrm{C}=\mathrm{O}$ str.), $1555 \mathrm{~cm}^{-1}$ (amide II, 
$\mathrm{N}-\mathrm{H}$ ben.), $1460 \mathrm{~cm}^{-1}$ (- $\mathrm{CH}_{2}-$ scissor); ${ }^{1} \mathrm{H}-\mathrm{NMR}\left(400 \mathrm{MHz}, \mathrm{CDCl}_{3}\right): \delta 0.878$ (t, terminal $\left.\mathrm{CH}_{3}, \quad 3 \mathrm{H}\right), \quad 1.282\left(-\mathrm{CH}_{3}\left(\mathrm{CH}_{2}\right)_{13} \mathrm{CH}_{2}-, 26 \mathrm{H}\right), 1.466 \quad\left(\mathrm{~s}, \mathrm{C}\left(\mathrm{CH}_{3}\right)_{3} \mathrm{OCO}-, \quad 9 \mathrm{H}\right), 1.549$ $\left(\mathrm{CH}_{3}\left(\mathrm{CH}_{2}\right)_{13} \mathrm{CH}_{2} \mathrm{CH}_{2}-, 2 \mathrm{H}\right), 3.2231\left(-\left(\mathrm{CH}_{3}\right) \mathrm{N}^{+}\left(\mathrm{CH}_{2} \mathrm{CH}_{2}\right)_{2}\left(\mathrm{CH}_{2} \mathrm{CONH}\right)-, 3 \mathrm{H}\right), 3.825-3.941$ ($\left.\left(\mathrm{CH}_{3}\right) \mathrm{N}^{+}\left(\mathrm{CH}_{2} \mathrm{CH}_{2}\right)_{2}\left(\mathrm{CH}_{2} \mathrm{CONH}\right)-, 4 \mathrm{H}\right), 4.580\left(-\mathrm{CH}_{2} \mathrm{CONHCH}_{2} \mathrm{CH}_{2}-, 2 \mathrm{H}\right), 5.012-5.236$ ($\left.\left(\mathrm{CH}_{3}\right) \mathrm{N}^{+}\left(\mathrm{CH}_{2} \mathrm{CH}_{2}\right)_{2}\left(\mathrm{CH}_{2} \mathrm{CONH}\right)-, 2 \mathrm{H}\right), 8.320\left(-\mathrm{CH}_{2} \mathrm{CONHCH}_{2} \mathrm{CH}_{2-}, 1 \mathrm{H}\right)$.

5c": FT-IR ( $\bar{v}): 3200-3450 \mathrm{~cm}^{-1}$ (amide N-H str.), $2940 \mathrm{~cm}^{-1}$ (- $\mathrm{CH}_{2}-$ assym. str.), $2855 \mathrm{~cm}^{-1}$ ( $-\mathrm{CH}_{2}-$ sym. str.), $1740 \mathrm{~cm}^{-1}$ ( $-\mathrm{C}=\mathrm{O}$ str.), $1672 \mathrm{~cm}^{-1}$ (amide $\mathrm{I}, \mathrm{C}=\mathrm{O}$ str.), $1550 \mathrm{~cm}^{-1}$ (amide II, $\mathrm{N}-\mathrm{H}$ ben.), $1462 \mathrm{~cm}^{-1}$ (- $\mathrm{CH}_{2}-$ scissor); ${ }^{1} \mathrm{H}-\mathrm{NMR}\left(400 \mathrm{MHz}, \mathrm{CDCl}_{3}\right): \delta 0.882(\mathrm{t}$, terminal $\left.\mathrm{CH}_{3}, 3 \mathrm{H}\right), 1.284\left(-\mathrm{CH}_{3}\left(\mathrm{CH}_{2}\right)_{13} \mathrm{CH}_{2}-, 26 \mathrm{H}\right), 1.459\left(\mathrm{~s}, \mathrm{C}\left(\mathrm{CH}_{3}\right)_{3} \mathrm{OCO}-\right), 1.548\left(\mathrm{CH}_{3}\left(\mathrm{CH}_{2}\right)_{13} \mathrm{CH}_{2-}\right.$ $\left.\mathrm{CH}_{2}-, \quad 2 \mathrm{H}\right), \quad 3.199 \quad\left(-\left(\mathrm{CH}_{3}\right) \mathrm{N}^{+}\left(\mathrm{CH}_{2} \mathrm{CH}_{2}\right)_{2}\left(\mathrm{CH}_{2} \mathrm{CONH}\right)-, \quad 3 \mathrm{H}\right), \quad 3.795-3.896 \quad(-$ $\left.\left(\mathrm{CH}_{3}\right) \mathrm{N}^{+}\left(\mathrm{CH}_{2} \mathrm{CH}_{2}\right)_{2}\left(\mathrm{CH}_{2} \mathrm{CONH}\right)-, 4 \mathrm{H}\right), 4.620\left(-\mathrm{CH}_{2} \mathrm{CONHCH}_{2} \mathrm{CH}_{2-}, 2 \mathrm{H}\right)$, 5.185-5.396 ($\left.\left(\mathrm{CH}_{3}\right) \mathrm{N}^{+}\left(\mathrm{CH}_{2} \mathrm{CH}_{2}\right)_{2}\left(\mathrm{CH}_{2} \mathrm{CONH}\right)-, 2 \mathrm{H}\right), 8.298\left(-\mathrm{CH}_{2} \mathrm{CONHCH}_{2} \mathrm{CH}_{2}-, 1 \mathrm{H}\right)$.

5a: FT-IR ( $\bar{v}$ ): 3200-3450 $\mathrm{cm}^{-1}$ (amide $\mathrm{N}-\mathrm{H}$ str.), $2942 \mathrm{~cm}^{-1}\left(-\mathrm{CH}_{2}-\right.$ assym. str.), $2855 \mathrm{~cm}^{-1}$ (- $\mathrm{CH}_{2}-$ sym. str.), $1670 \mathrm{~cm}^{-1}$ (amide $\mathrm{I}, \mathrm{C}=\mathrm{O}$ str.), $1639 \mathrm{~cm}^{-1}$ (ester $-\mathrm{C}=\mathrm{O}$ str.), $1555 \mathrm{~cm}^{-1}$ (amide II, N-H ben.), $1460 \mathrm{~cm}^{-1}\left(-\mathrm{CH}_{2}-\right.$ scissor); ${ }^{1} \mathrm{H}-\mathrm{NMR}\left(400 \mathrm{MHz}, \mathrm{CDCl}_{3}\right): \delta 0.881$ (t, terminal $\left.-\mathrm{CH}_{3}, 3 \mathrm{H}\right), 1.284\left(-\mathrm{CH}_{3}\left(\mathrm{CH}_{2}\right)_{13} \mathrm{CH}_{2}-, 26 \mathrm{H}\right), 1.551\left(\mathrm{CH}_{3}\left(\mathrm{CH}_{2}\right)_{13} \mathrm{CH}_{2} \mathrm{CH}_{2}-, 2 \mathrm{H}\right), 3.230$ $\left(-\left(\mathrm{CH}_{3}\right) \mathrm{N}^{+}\left(\mathrm{CH}_{2} \mathrm{CH}_{2}\right)_{2}\left(\mathrm{CH}_{2} \mathrm{CONH}\right)-, 3 \mathrm{H}\right), 3.845-3.876\left(-\left(\mathrm{CH}_{3}\right) \mathrm{N}^{+}\left(\mathrm{CH}_{2} \mathrm{CH}_{2}\right)_{2}\left(\mathrm{CH}_{2} \mathrm{COO}\right)-\right.$ and $\left.\left(\mathrm{CH}_{3}\right) \mathrm{N}^{+}\left(\mathrm{CH}_{2} \mathrm{CH}_{2}\right)_{2}\left(\mathrm{CH}_{2} \mathrm{CONH}\right)-, 6 \mathrm{H}\right), 4.588\left(-\mathrm{CH}_{2} \mathrm{CONHCH}_{2} \mathrm{CH}_{2}-, 2 \mathrm{H}\right)$, 5.185-5.146 ($\left.\left(\mathrm{CH}_{3}\right) \mathrm{N}^{+}\left(\mathrm{CH}_{2} \mathrm{CH}_{2}\right)_{2}\left(\mathrm{CH}_{2} \mathrm{CONH}\right)-, 2 \mathrm{H}\right), 8.309\left(-\mathrm{CH}_{2} \mathrm{CONHCH}_{2} \mathrm{CH}_{2}-, 1 \mathrm{H}\right)$.

5b: FT-IR ( $\bar{v})$ : 3200-3450 $\mathrm{cm}^{-1}$ (amide $\mathrm{N}-\mathrm{H}$ str.), $2940 \mathrm{~cm}^{-1}\left(-\mathrm{CH}_{2}-\right.$ assym. str.), $2853 \mathrm{~cm}^{-1}$ ( $-\mathrm{CH}_{2}-$ sym. str.), $1671 \mathrm{~cm}^{-1}$ (amide I, $\mathrm{C}=\mathrm{O}$ str.), $1637 \mathrm{~cm}^{-1}$ (ester $-\mathrm{C}=\mathrm{O}$ str.), $1551 \mathrm{~cm}^{-1}$ (amide II, N-H ben.), $1460 \mathrm{~cm}^{-1}$ (- $\mathrm{CH}_{2}-$ scissor); ${ }^{1} \mathrm{H}-\mathrm{NMR}\left(400 \mathrm{MHz}, \mathrm{CDCl}_{3}\right): \delta 0.880(\mathrm{t}$, terminal $\left.-\mathrm{CH}_{3}, 3 \mathrm{H}\right), 1.284\left(-\mathrm{CH}_{3}\left(\mathrm{CH}_{2}\right)_{13} \mathrm{CH}_{2}-, 26 \mathrm{H}\right), 1.551\left(\mathrm{CH}_{3}\left(\mathrm{CH}_{2}\right)_{13} \mathrm{CH}_{2} \mathrm{CH}_{2}-, 2 \mathrm{H}\right), 3.229$ $\left(-\left(\mathrm{CH}_{3}\right) \mathrm{N}^{+}\left(\mathrm{CH}_{2} \mathrm{CH}_{2}\right)_{2}\left(\mathrm{CH}_{2} \mathrm{CONH}\right)-, 3 \mathrm{H}\right), 3.835-3.976\left(-\left(\mathrm{CH}_{3}\right) \mathrm{N}^{+}\left(\mathrm{CH}_{2} \mathrm{CH}_{2}\right)_{2}\left(\mathrm{CH}_{2} \mathrm{COO}\right)-\right.$ and $\left.\left(\mathrm{CH}_{3}\right) \mathrm{N}^{+}\left(\mathrm{CH}_{2} \mathrm{CH}_{2}\right)_{2}\left(\mathrm{CH}_{2} \mathrm{CONH}\right)-, 6 \mathrm{H}\right), 4.600\left(-\mathrm{CH}_{2} \mathrm{CONHCH}_{2} \mathrm{CH}_{2}-, 2 \mathrm{H}\right)$, 5.025-5.046 ($\left.\left(\mathrm{CH}_{3}\right) \mathrm{N}^{+}\left(\mathrm{CH}_{2} \mathrm{CH}_{2}\right)_{2}\left(\mathrm{CH}_{2} \mathrm{CONH}\right)-, 2 \mathrm{H}\right), 8.329\left(-\mathrm{CH}_{2} \mathrm{CONHCH}_{2} \mathrm{CH}_{2}-, 1 \mathrm{H}\right)$.

5c: FT-IR ( $\bar{v})$ : 3200-3450 $\mathrm{cm}^{-1}$ (amide $\mathrm{N}-\mathrm{H}$ str.), $2945 \mathrm{~cm}^{-1}\left(-\mathrm{CH}_{2}-\right.$ assym. str.), $2855 \mathrm{~cm}^{-1}$ ( $-\mathrm{CH}_{2}-$ sym. str.), $1670 \mathrm{~cm}^{-1}$ (amide I, $\mathrm{C}=\mathrm{O}$ str.), $1635 \mathrm{~cm}^{-1}$ (ester $-\mathrm{C}=\mathrm{O}$ str.), $1554 \mathrm{~cm}^{-1}$ (amide II, N-H ben.), $1462 \mathrm{~cm}^{-1}$ (- $\mathrm{CH}_{2}-$ scissor); ${ }^{1} \mathrm{H}-\mathrm{NMR}\left(400 \mathrm{MHz}, \mathrm{CDCl}_{3}\right): \delta 0.884$ (t, terminal $\left.-\mathrm{CH}_{3}, 3 \mathrm{H}\right), 1.289\left(-\mathrm{CH}_{3}\left(\mathrm{CH}_{2}\right)_{13} \mathrm{CH}_{2}-, 26 \mathrm{H}\right), 1.548\left(\mathrm{CH}_{3}\left(\mathrm{CH}_{2}\right)_{13} \mathrm{CH}_{2} \mathrm{CH}_{2}-, 2 \mathrm{H}\right), 3.232$ $\left(-\left(\mathrm{CH}_{3}\right) \mathrm{N}^{+}\left(\mathrm{CH}_{2} \mathrm{CH}_{2}\right)_{2}\left(\mathrm{CH}_{2} \mathrm{CONH}\right)-, 3 \mathrm{H}\right), 3.795-3.989\left(-\left(\mathrm{CH}_{3}\right) \mathrm{N}^{+}\left(\mathrm{CH}_{2} \mathrm{CH}_{2}\right)_{2}\left(\mathrm{CH}_{2} \mathrm{COO}\right)-\right.$ and $\left.\left(\mathrm{CH}_{3}\right) \mathrm{N}^{+}\left(\mathrm{CH}_{2} \mathrm{CH}_{2}\right)_{2}\left(\mathrm{CH}_{2} \mathrm{CONH}\right)-, 6 \mathrm{H}\right), 4.619\left(-\mathrm{CH}_{2} \mathrm{CONHCH}_{2} \mathrm{CH}_{2}-, 2 \mathrm{H}\right)$, 5.187-5.141 ($\left.\left(\mathrm{CH}_{3}\right) \mathrm{N}^{+}\left(\mathrm{CH}_{2} \mathrm{CH}_{2}\right)_{2}\left(\mathrm{CH}_{2} \mathrm{CONH}\right)-, 2 \mathrm{H}\right), 8.304\left(-\mathrm{CH}_{2} \mathrm{CONHCH}_{2} \mathrm{CH}_{2}-, 1 \mathrm{H}\right)$.

5d': FT-IR ( $\bar{v}): 2940 \mathrm{~cm}^{-1}$ (- $\mathrm{CH}_{2}-$ assym. str.), $2853 \mathrm{~cm}^{-1}\left(-\mathrm{CH}_{2}-\right.$ sym. str.), $1737 \mathrm{~cm}^{-1}$ $\left(-\mathrm{C}=\mathrm{O}\right.$ str.), $1460 \mathrm{~cm}^{-1} \quad\left(-\mathrm{CH}_{2}-\right.$ scissor $) ;{ }^{1} \mathrm{H}-\mathrm{NMR}\left(400 \mathrm{MHz}, \mathrm{CDCl}_{3}\right): \delta 1.456$ (s, $\left.\mathrm{C}\left(\mathrm{CH}_{3}\right)_{3} \mathrm{OCO}-, 9 \mathrm{H}\right), 3.229\left(-\left(\mathrm{CH}_{3}\right) \mathrm{N}^{+}\left(\mathrm{CH}_{2} \mathrm{CH}_{2}\right)-, 3 \mathrm{H}\right), 3.835-3.976\left(-\left(\mathrm{CH}_{3}\right) \mathrm{N}^{+}\left(\mathrm{CH}_{2} \mathrm{CH}_{2}\right)-\right.$, $4 \mathrm{H}), 4.600\left(-\mathrm{CH}_{2} \mathrm{COO}-, 2 \mathrm{H}\right)$.

5d: FT-IR ( $\bar{v}): 2948 \mathrm{~cm}^{-1}$ (- $\mathrm{CH}_{2}-$ assym. str.), $2873 \mathrm{~cm}^{-1}\left(-\mathrm{CH}_{2}-\right.$ sym. str.), $1640 \mathrm{~cm}^{-1}$ (-C=O str.), $1468 \mathrm{~cm}^{-1}$ (- $\mathrm{CH}_{2}-$ scissor); ${ }^{1} \mathrm{H}-\mathrm{NMR}\left(400 \mathrm{MHz}, \mathrm{CDCl}_{3}\right): \delta 3.039-3.210(-$ $\left.\left(\mathrm{CH}_{3}\right) \mathrm{N}^{+}\left(\mathrm{CH}_{2} \mathrm{CH}_{2}\right)-, 3 \mathrm{H}\right), 3.435-3.876\left(-\left(\mathrm{CH}_{3}\right) \mathrm{N}^{+}\left(\mathrm{CH}_{2} \mathrm{CH}_{2}\right)-, 4 \mathrm{H}\right), 4.044\left(-\mathrm{CH}_{2} \mathrm{COO}-, 2 \mathrm{H}\right)$. 


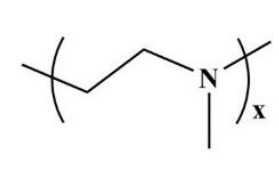

$N$-Methyl PEI

$(\mathrm{PEI}=22 \mathrm{kDa})$

\section{$\left(\mathrm{Me}_{3}\right) \mathrm{COCOCH}_{2} \mathrm{Br}$,}

$\mathrm{CHCl}_{3}, 55{ }^{\circ} \mathrm{C}(72 \mathrm{~h})$

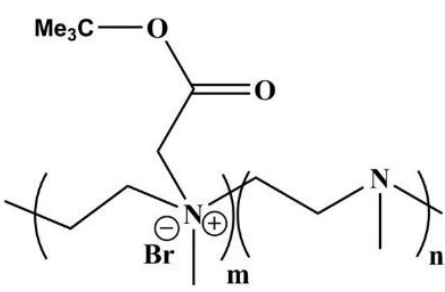

Partially quaternized $N$-methyl PEI $\left(\mathrm{DQ}=22 \%\left(\mathbf{5} \mathbf{a}^{\prime}\right), 48 \%\left(\mathbf{5} \mathbf{b}^{\prime}\right)\right.$ and $\left.78 \%\left(\mathbf{5} \mathbf{c}^{\prime}\right)\right)$ $\mathrm{C}_{16} \mathrm{H}_{33} \mathrm{NHCOCH}_{2} \mathrm{Br}$, $\mathrm{CHCl}_{3}, 85 \mathrm{C}(96 \mathrm{~h})$

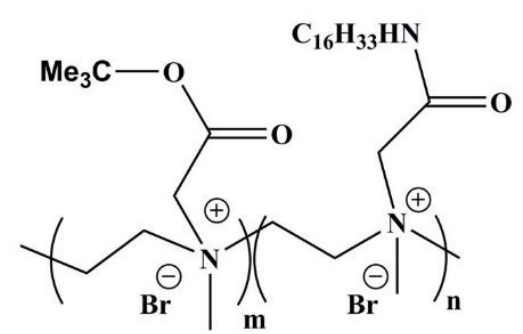

Quaternized $N$-methyl PEI $(\mathrm{m}=22 \%$ $\left(\mathbf{5} \mathbf{a}^{\prime \prime}\right), 48 \%\left(\mathbf{5} \mathbf{b}^{\prime \prime}\right)$ and $\left.78 \%\left(\mathbf{5} \mathbf{c}^{\prime \prime}\right)\right)$

Partially zwitterionic quaternary PEIs (zwitterionic moiety $=22 \%(5 a)$, $48 \%(\mathbf{5 b})$ and $78 \%(\mathbf{5 c}))$

Scheme S1. Synthesis of partially zwitterionic PEI derivatives. 


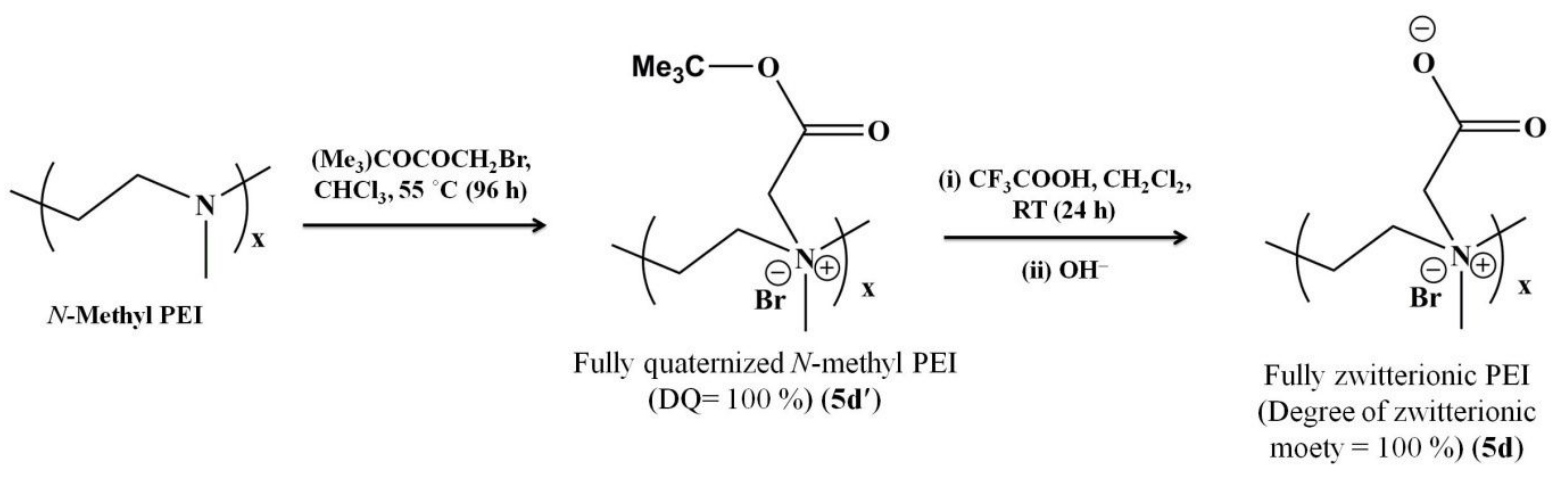

Scheme S2. Synthesis of partially zwitterionic PEI derivative (5d). 


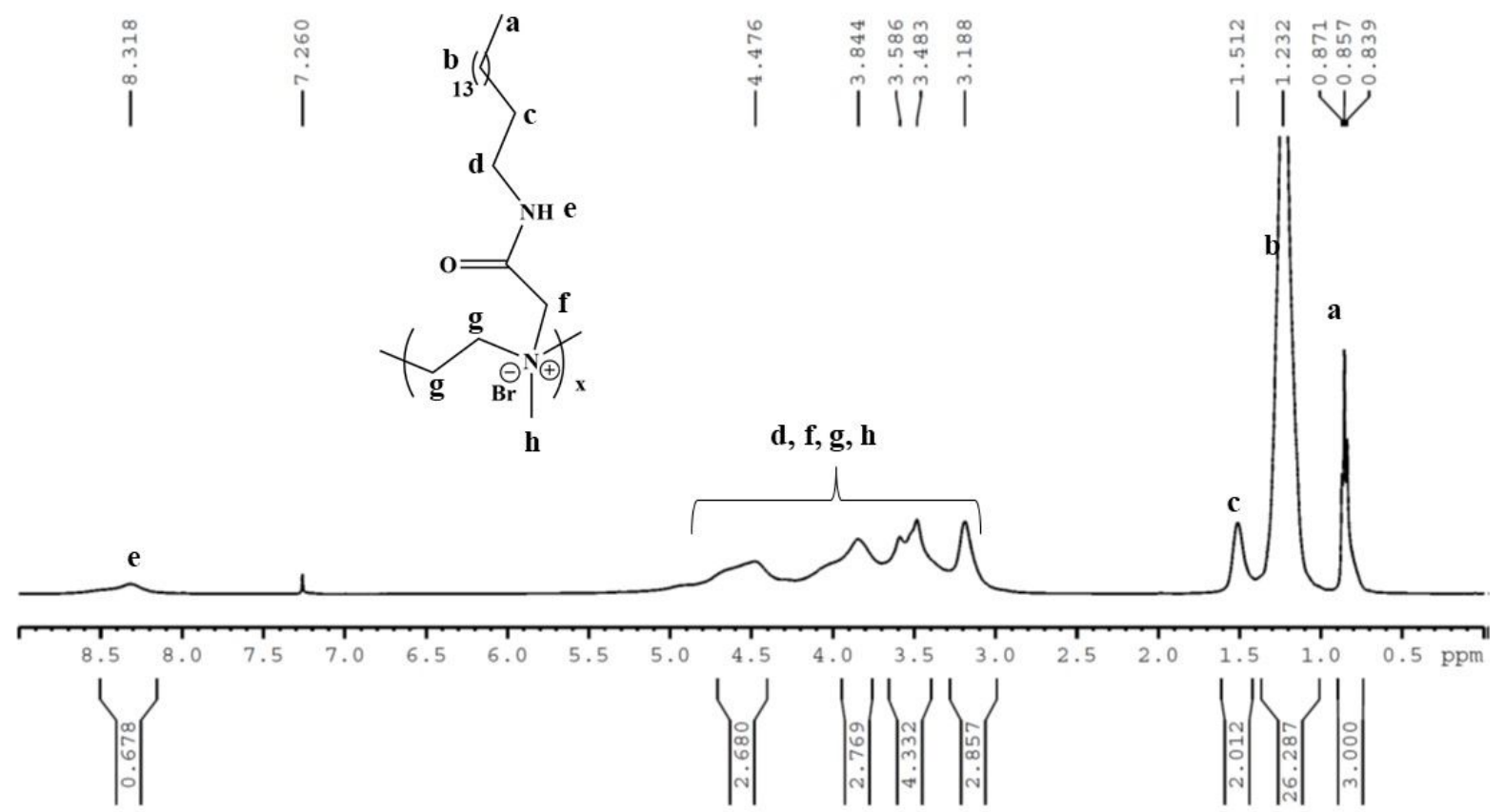

Figure S1. ${ }^{1} \mathrm{H}-\mathrm{NMR}$ spectrum of $\mathbf{1 a}$. 


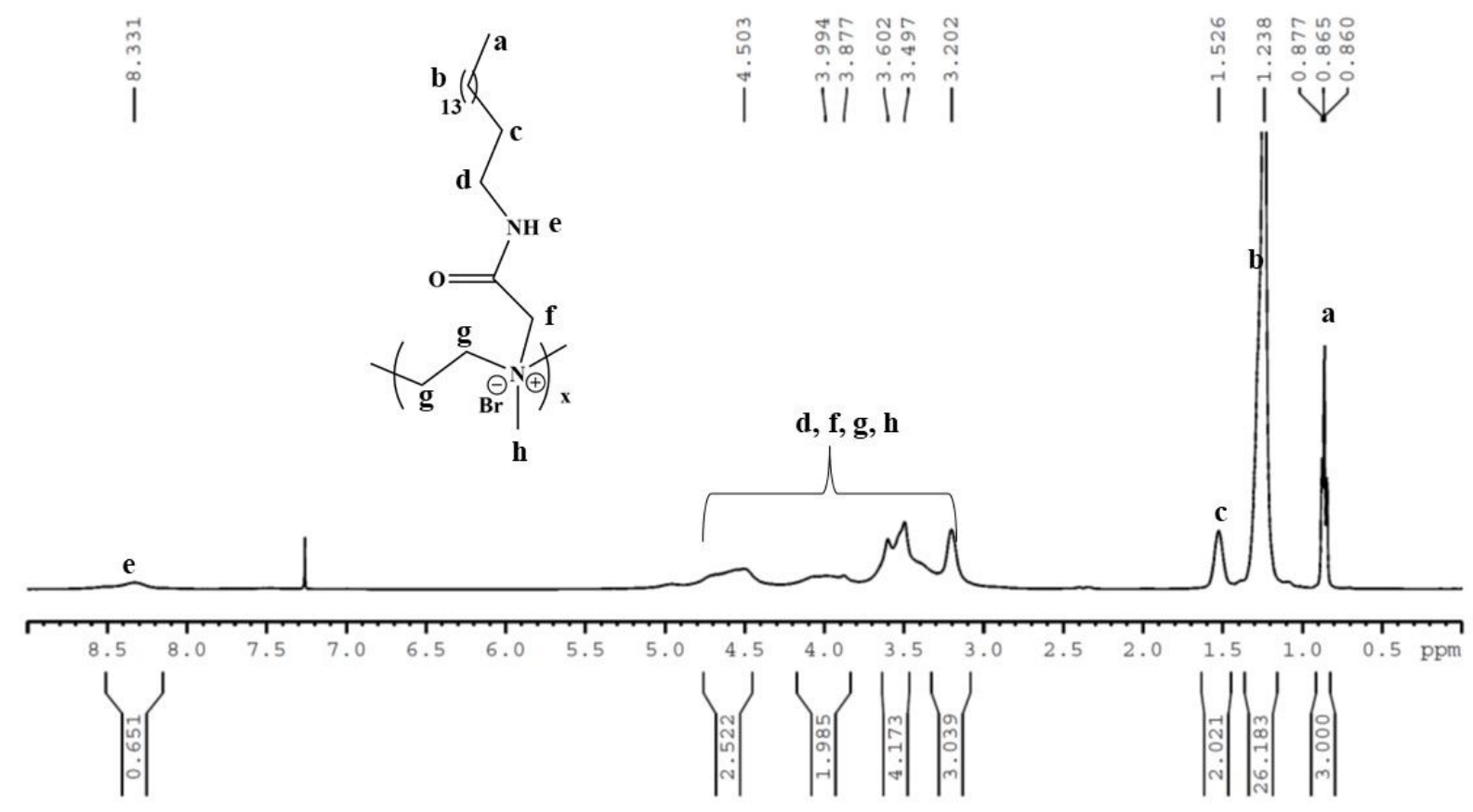

Figure S2. ${ }^{1} \mathrm{H}-\mathrm{NMR}$ spectrum of $\mathbf{1 b}$. 


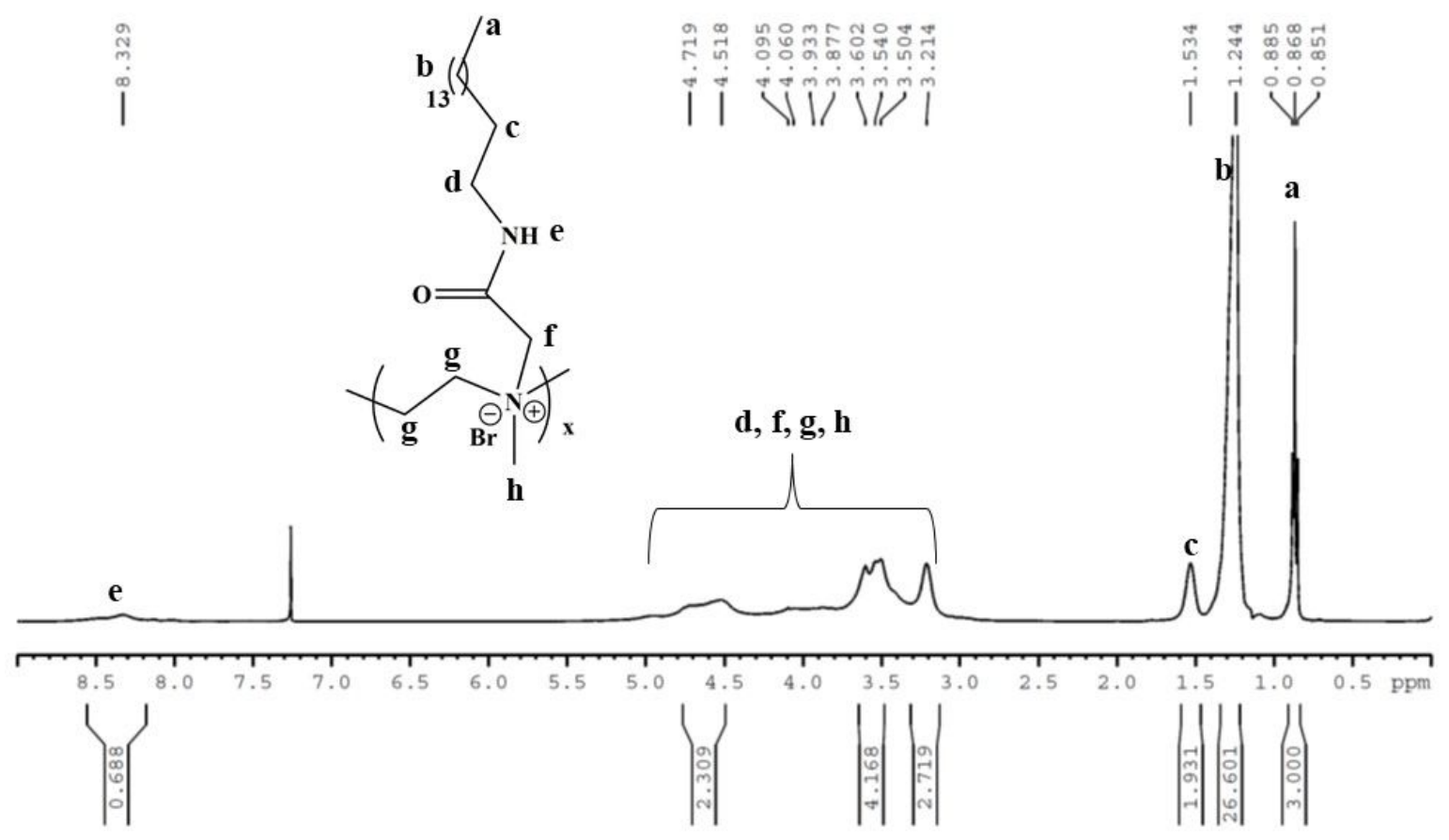

Figure S3. ${ }^{1} \mathrm{H}-\mathrm{NMR}$ spectrum of $\mathbf{1 c}$. 


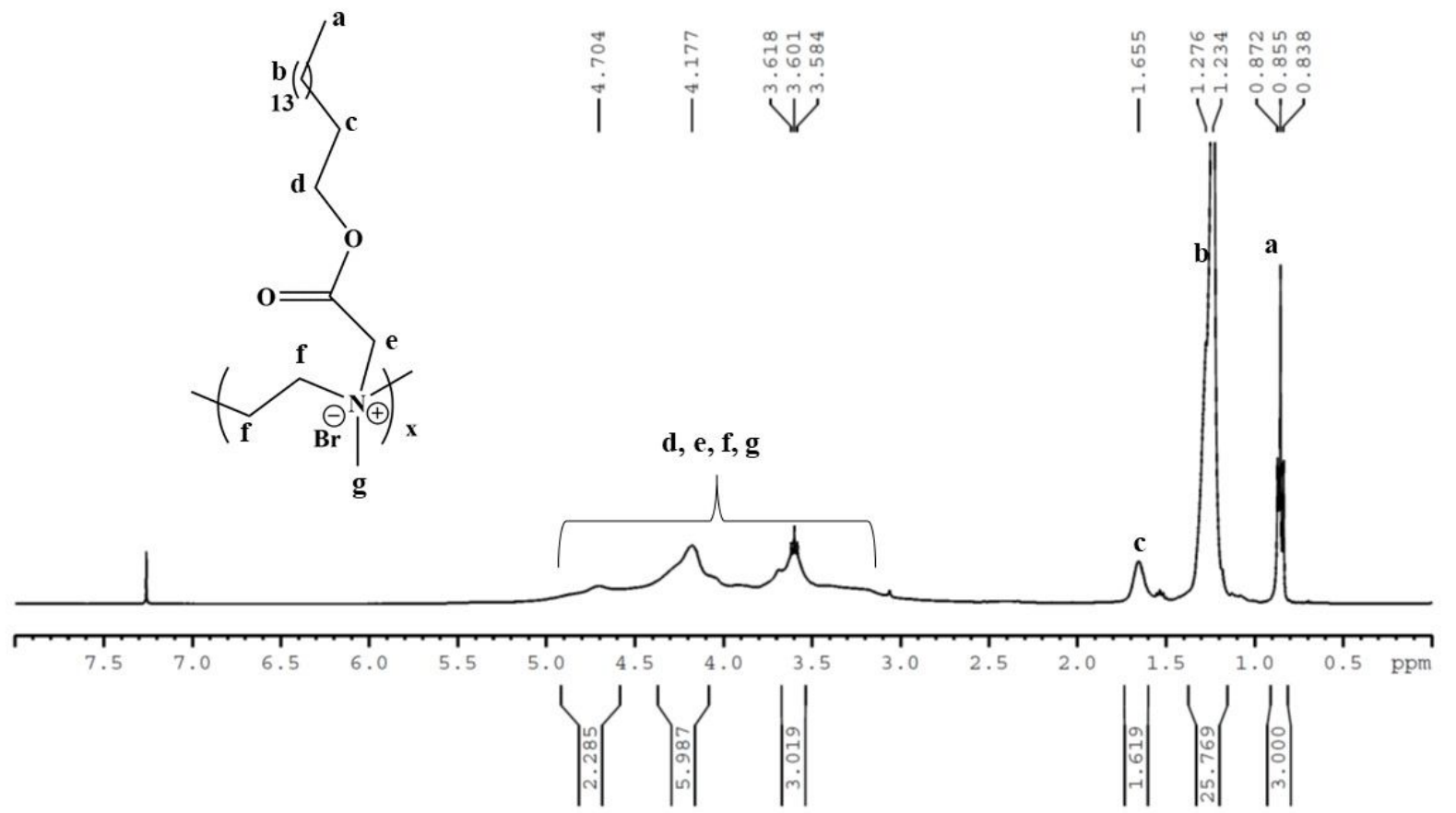

Figure S4. ${ }^{1} \mathrm{H}-\mathrm{NMR}$ spectrum of $\mathbf{2 a}$. 


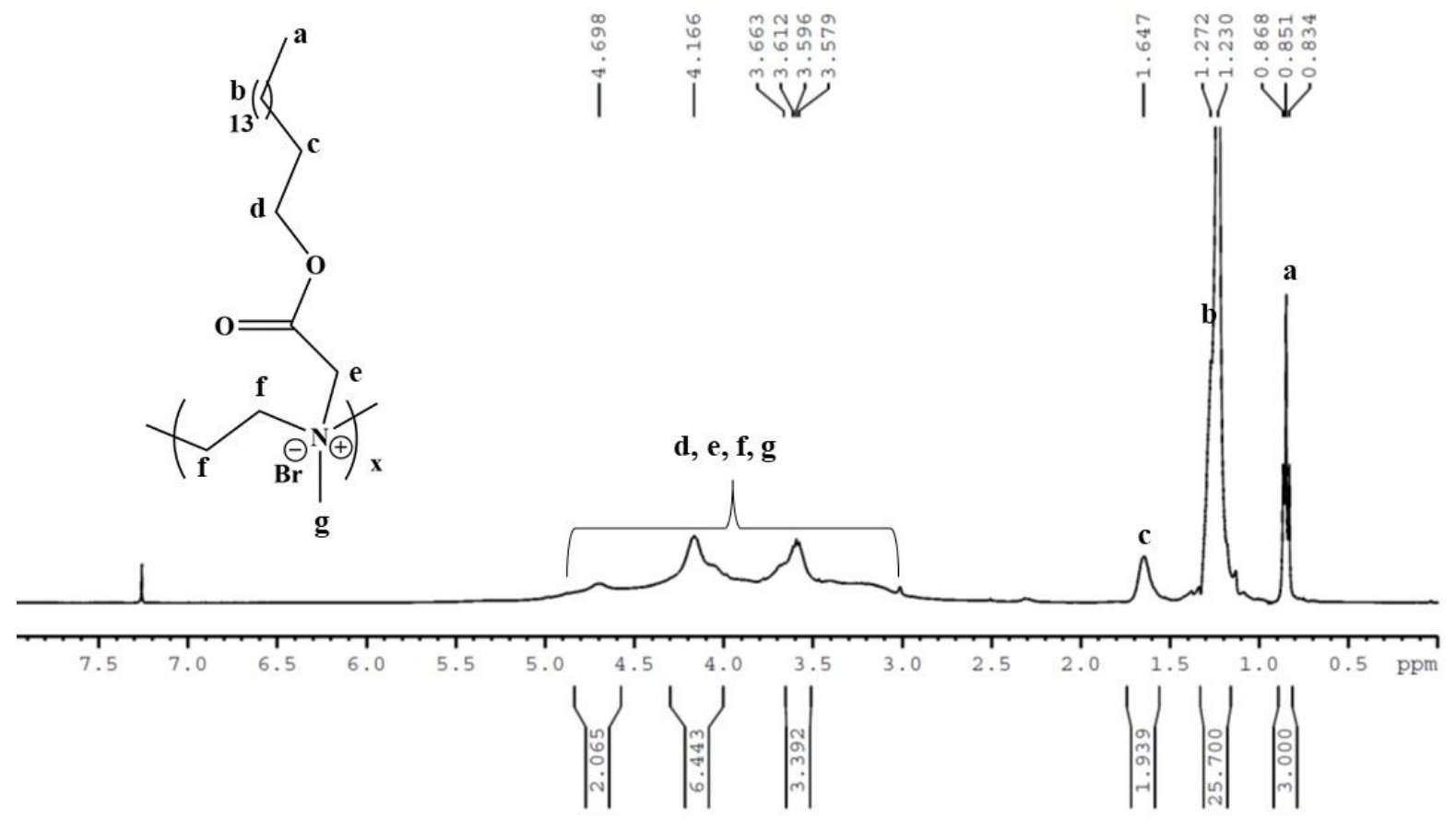

Figure S5. ${ }^{1} \mathrm{H}-\mathrm{NMR}$ spectrum of $\mathbf{2} \mathbf{b}$. 


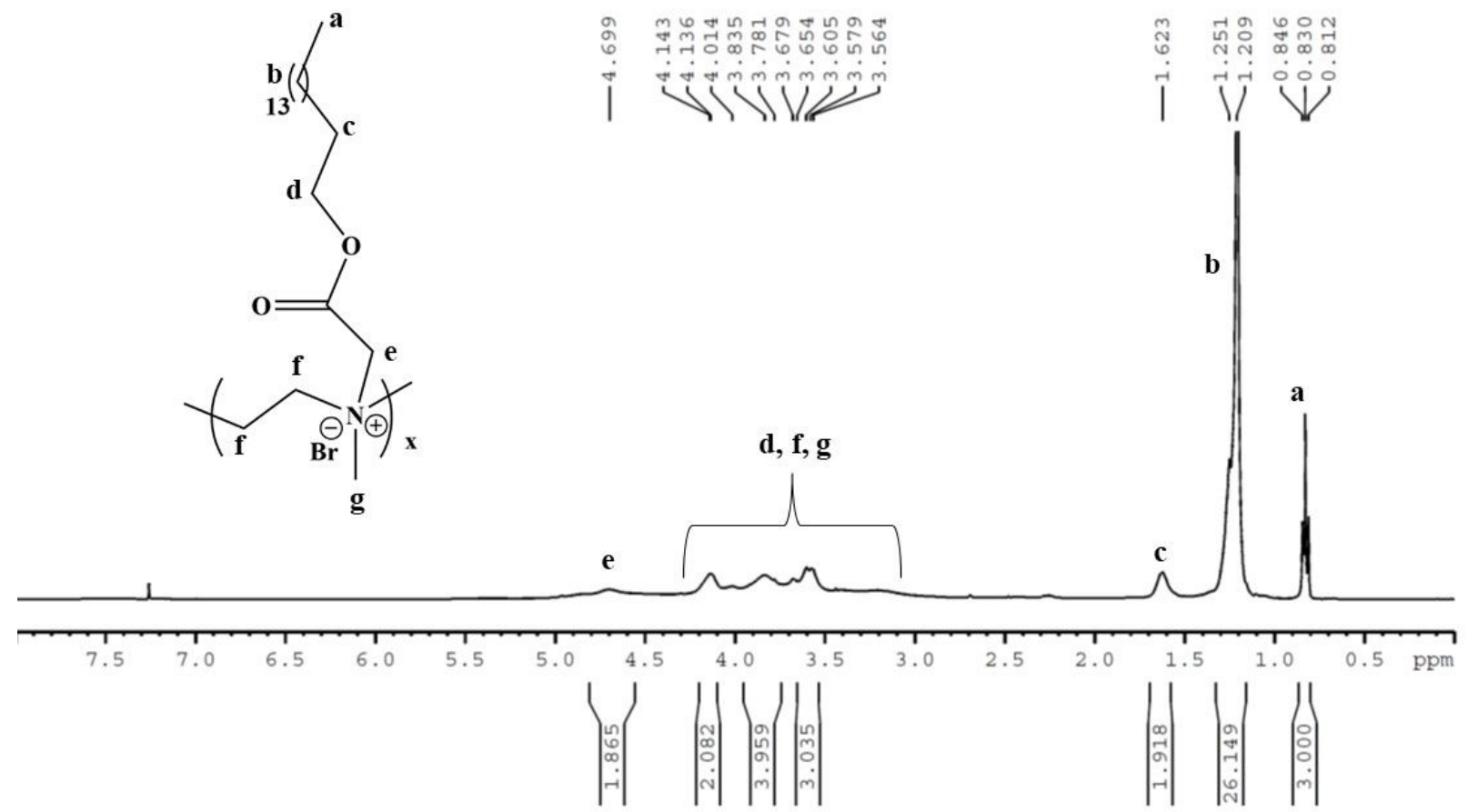

Figure S6. ${ }^{1} \mathrm{H}-\mathrm{NMR}$ spectrum of $\mathbf{2 c}$. 


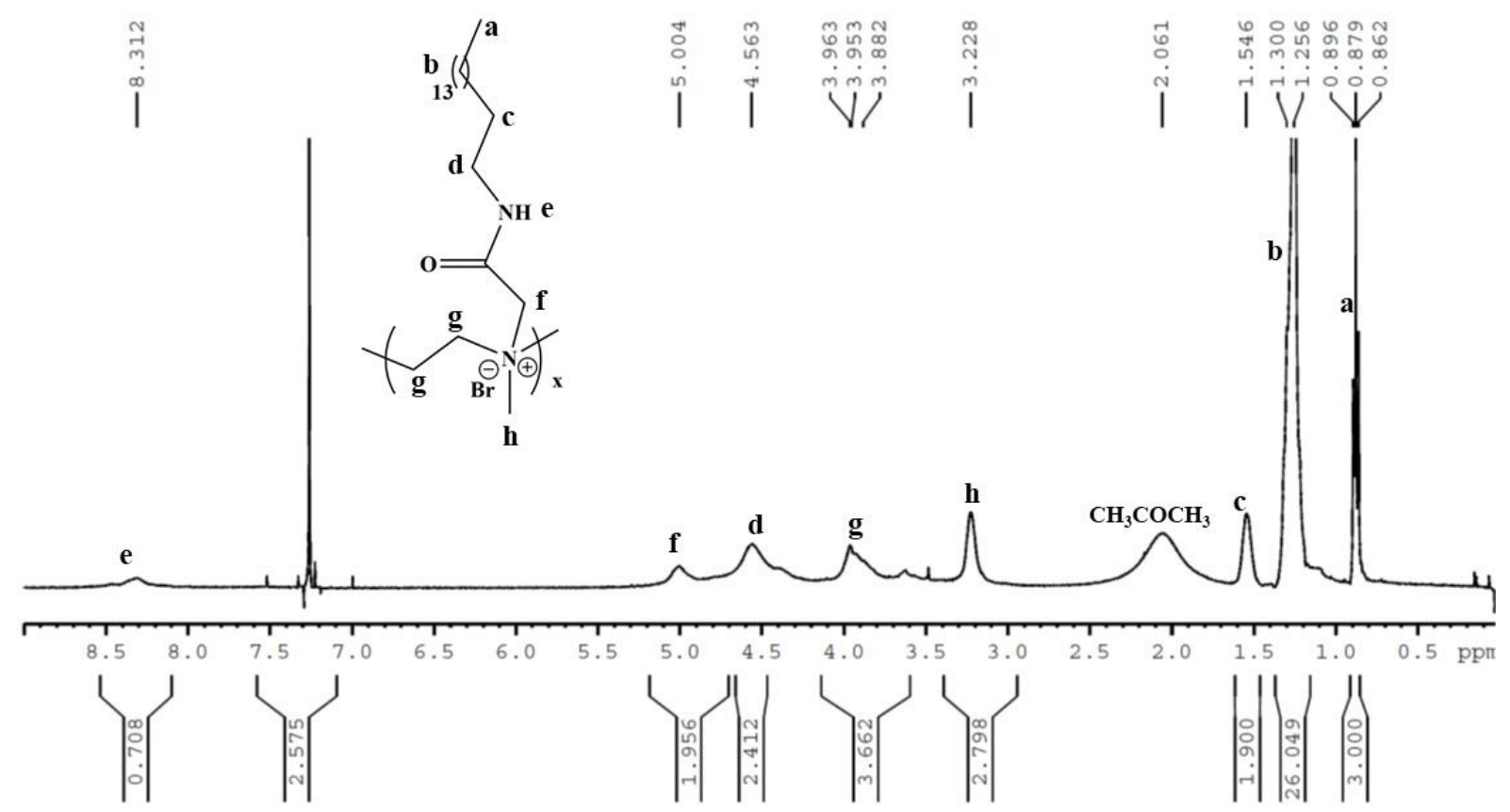

Figure S7. ${ }^{1} \mathrm{H}-\mathrm{NMR}$ spectrum of $\mathbf{3 a}$. 


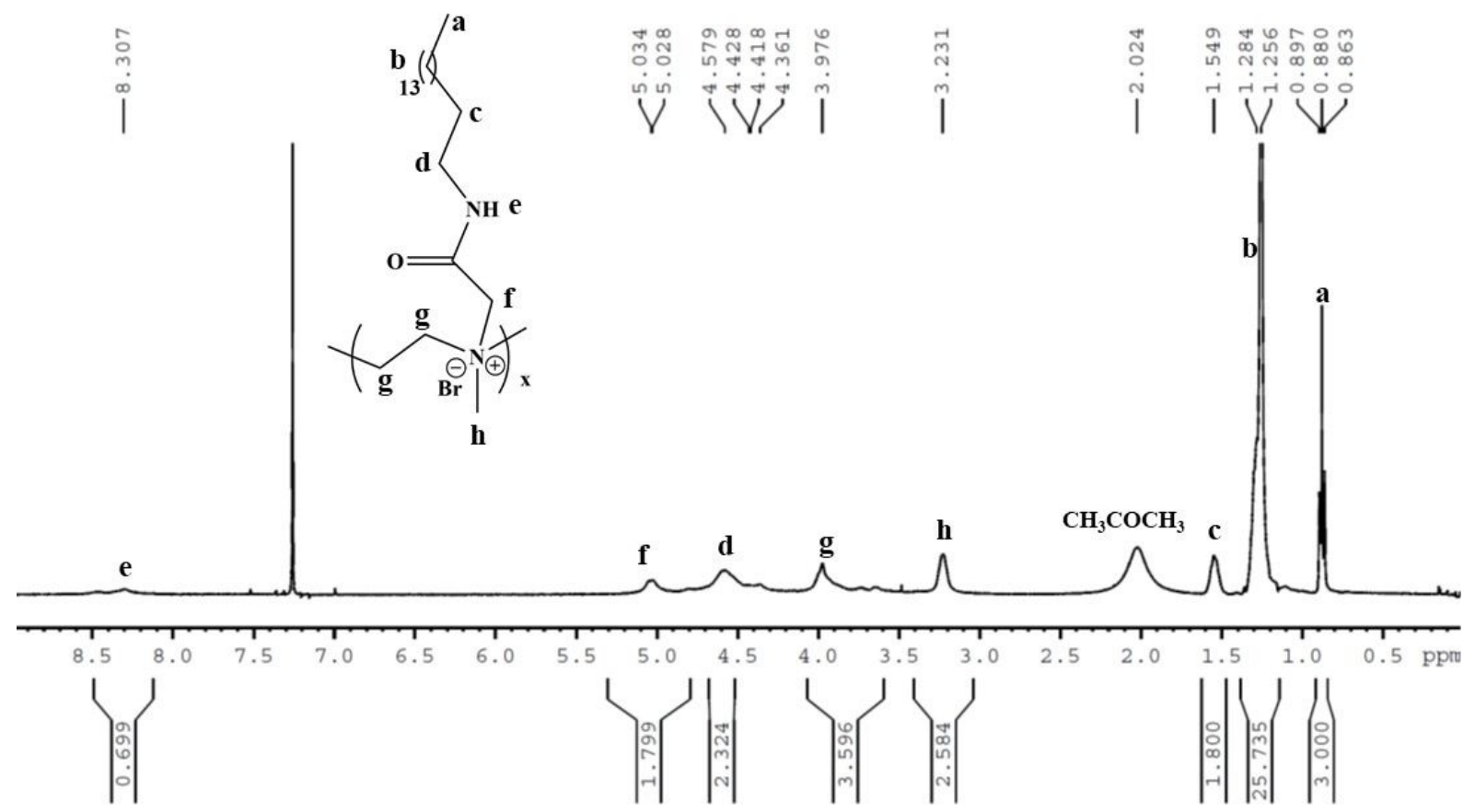

Figure S8. ${ }^{1} \mathrm{H}-\mathrm{NMR}$ spectrum of $\mathbf{3 b}$. 


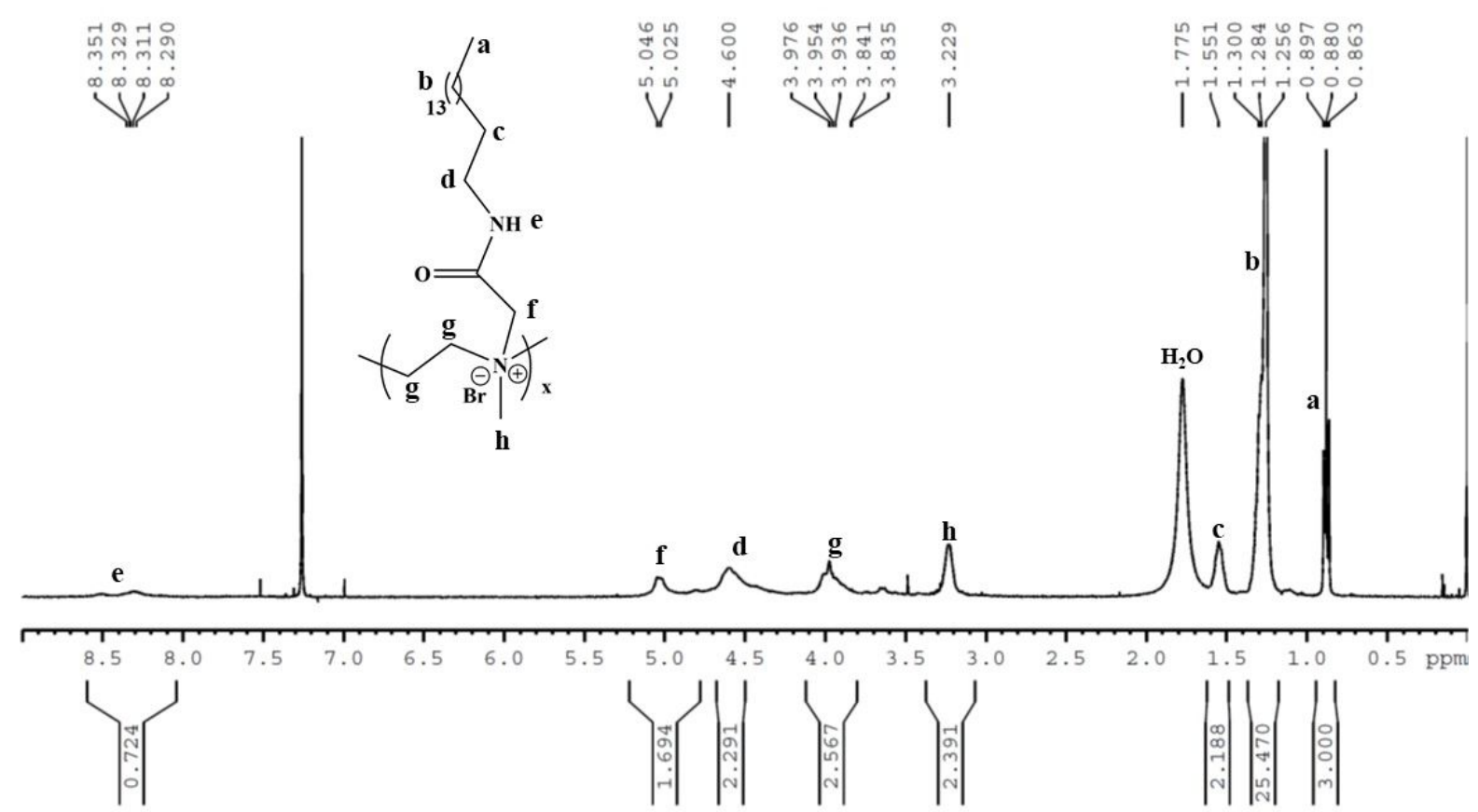

Figure S9. ${ }^{1} \mathrm{H}-\mathrm{NMR}$ spectrum of $3 \mathbf{c}$. 


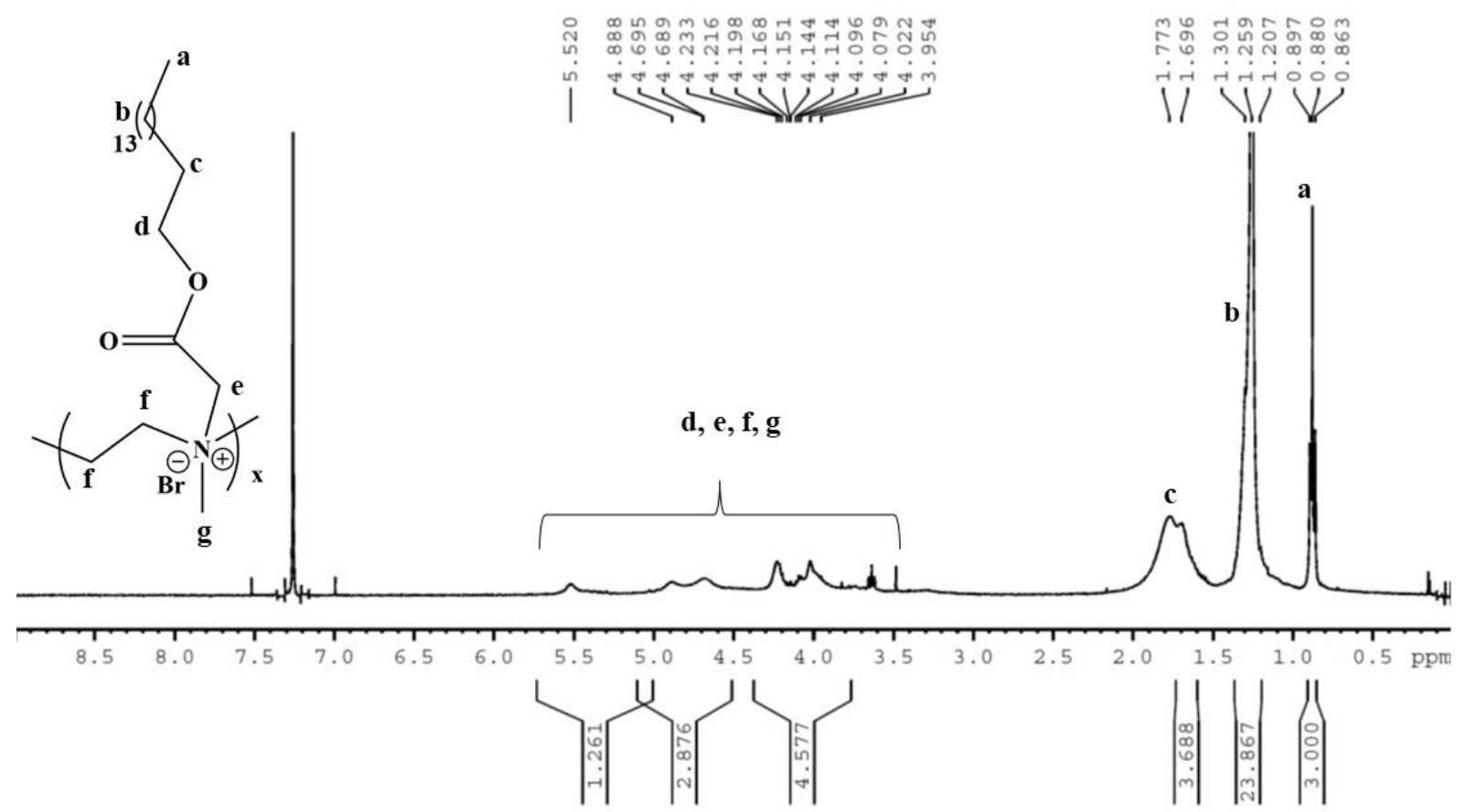

Figure S10. ${ }^{1} \mathrm{H}-\mathrm{NMR}$ spectrum of $\mathbf{4 a}$. 


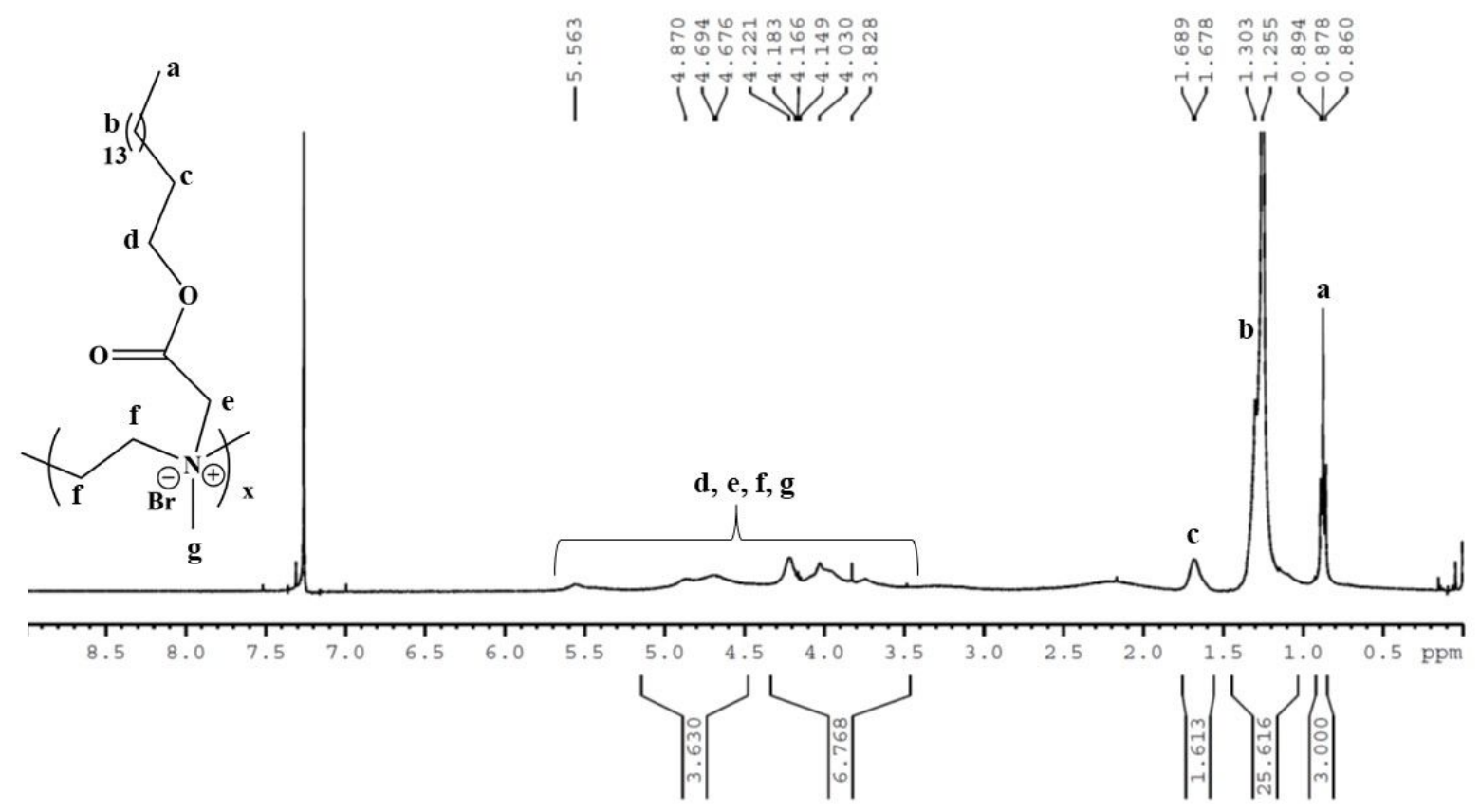

Figure S11. ${ }^{1} \mathrm{H}-\mathrm{NMR}$ spectrum of $\mathbf{4 b}$. 


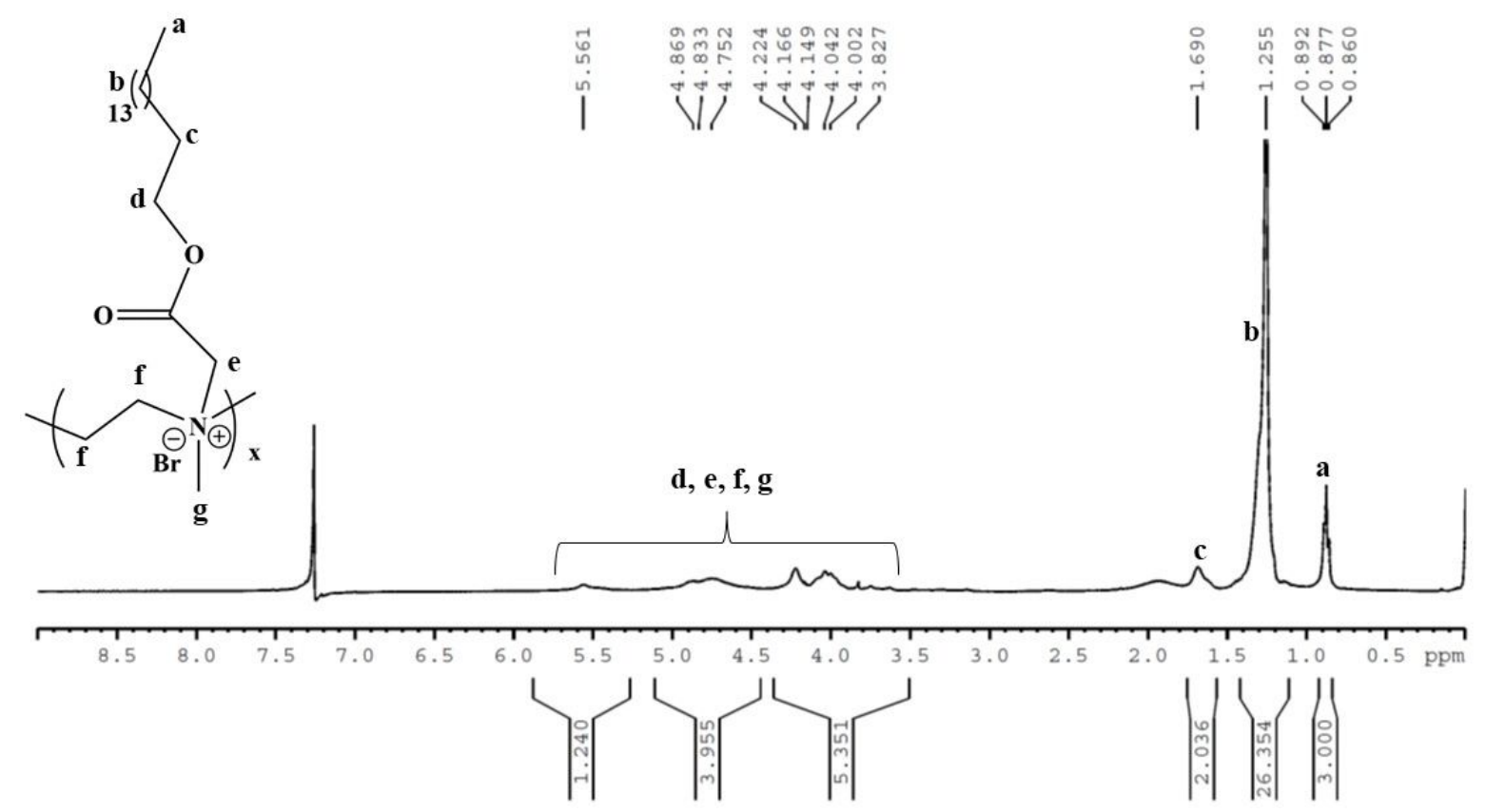

Figure S12. ${ }^{1} \mathrm{H}-\mathrm{NMR}$ spectrum of $\mathbf{4 c}$. 


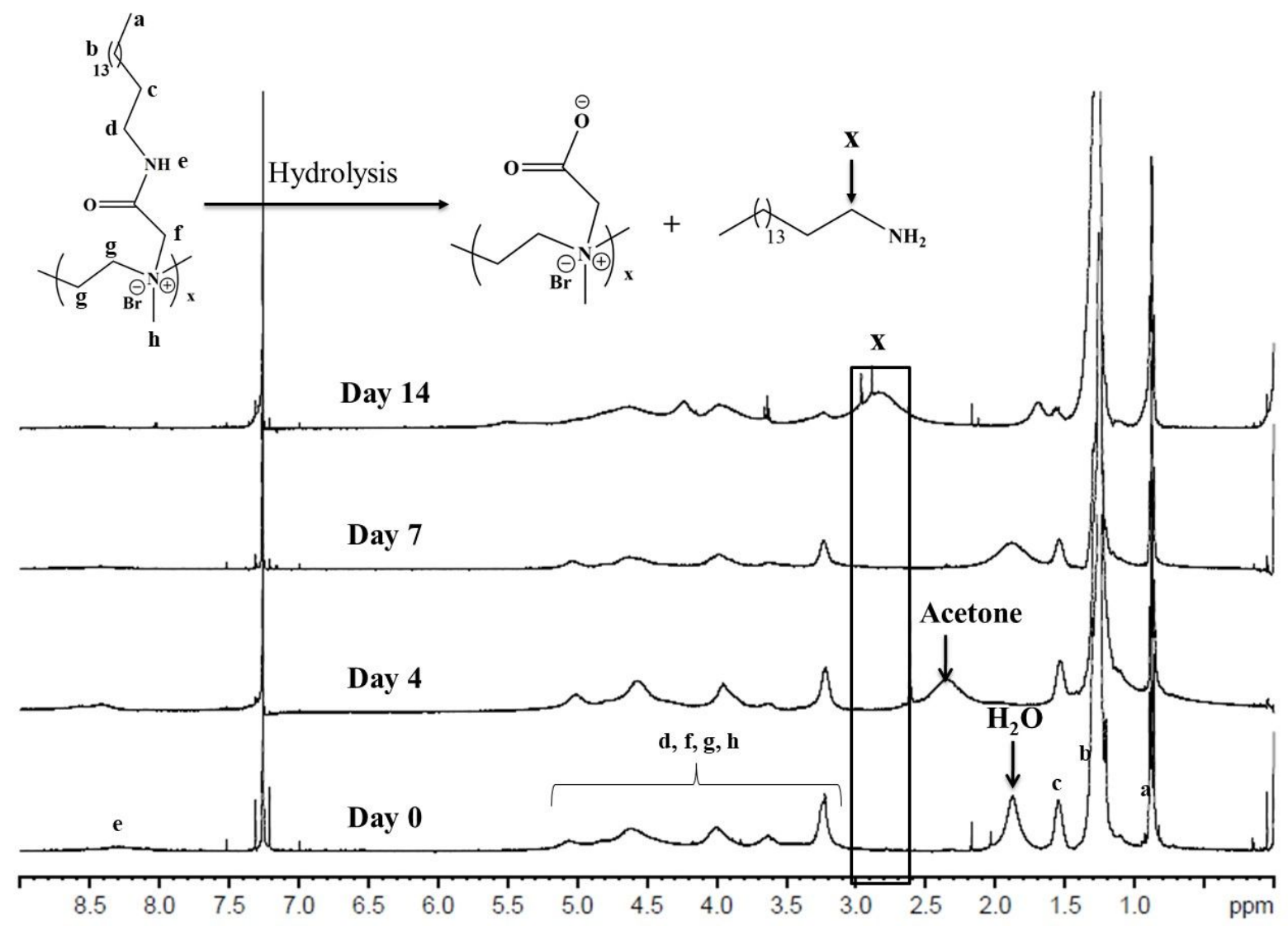

Figure S13 Representative ${ }^{1} \mathrm{H}-\mathrm{NMR}$ spectra of $\mathbf{3 c}$ during acidic treatment in the presence of $\mathrm{HCl}$ at different time periods. In the ${ }^{1} \mathrm{H}-\mathrm{NMR}$ spectra, ' $\mathrm{x}$ ' represents $\alpha-\mathrm{CH}_{2}$ protons of hydrolyzed product 1-hexadecylamine (protonated under the experimental condition). 

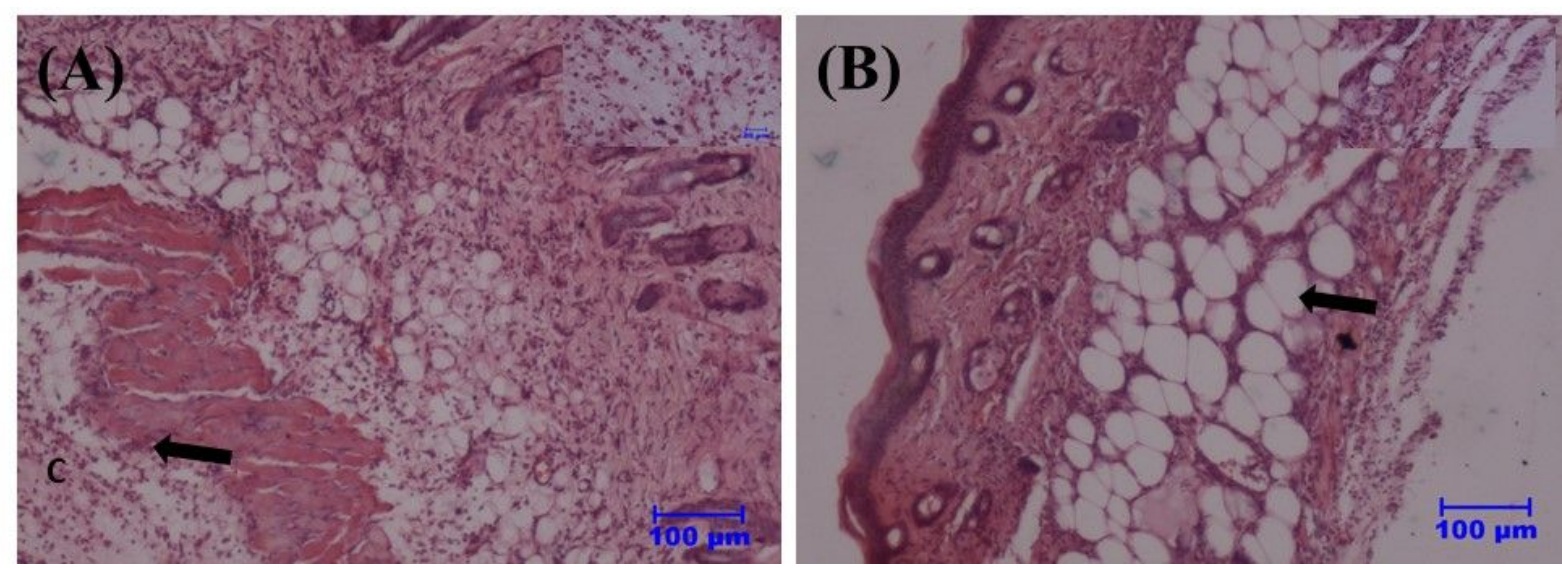

Figure S14. In-vivo antibacterial activity of the cationic PEI derivatives. Mice skin and subcutaneous tissue histopathology: (A) tissue surrounding MRSA-infected catheter coated with (F) $4 \mu \mathrm{g} / \mathrm{mm}^{2}$ of 3c, (B) $4 \mu \mathrm{g} / \mathrm{mm}^{2}$ of $4 \mathbf{c}$. Cells were stained with haematoxylin and eosin staining agents. Insets are the high resolution images. 